\title{
REGULARITY FOR FULLY NONLINEAR INTEGRO-DIFFERENTIAL OPERATORS WITH REGULARLY VARYING KERNELS
}

\author{
SOOJUNG KIM, YONG-CHEOL KIM, AND KI-AHM LEE
}

\begin{abstract}
AвSTRACT. In this paper, the regularity results for the integro-differential operators of the fractional Laplacian type by Caffarelli and Silvestre [CS1] are extended to those for the integro-differential operators associated with symmetric, regularly varying kernels at zero. In particular, we obtain the uniform Harnack inequality and Hölder estimate of viscosity solutions to the nonlinear integro-differential equations associated with the kernels $K_{\sigma, \beta}$ satisfying

$$
K_{\sigma, \beta}(y) \asymp \frac{2-\sigma}{|y|^{n+\sigma}}\left(\log \frac{2}{|y|^{2}}\right)^{\beta(2-\sigma)} \quad \text { near zero }
$$

with respect to $\sigma \in(0,2)$ close to 2 (for a given $\beta \in \mathbb{R}$ ), where the regularity estimates do not blow up as the order $\sigma \in(0,2)$ tends to 2 .
\end{abstract}

\section{Contents}

1. Introduction

1.1. Introduction

1.2. Integro-differential operators

1.3. Main results

2. Viscosity solutions

3. Regularity estimates for integro-differential operators with regularly varying kernels

3.1. Aleksandrov-Bakelman-Pucci type estimate 14

3.2. Barrier function 16

3.3. Power decay estimate of super-level sets 19

3.4. Harnack inequality 22

3.5. Hölder continuity 25

3.6. $C^{1, \alpha}$ estimate 25

3.7. Truncated kernels at infinity 26

4. Uniform regularity estimates for certain integro-differential operators as $\sigma \rightarrow 2-$

Appendix A. Regular variations $\quad 29$

References

\section{InTRODUCTION}

1.1. Introduction. In this paper, we are concerned with fully nonlinear elliptic integrodifferential operators associated with symmetric, regularly varying kernels at zero. From the Lévy-Khinchine formula, the purely jump processes which allow particles to interact 
at large scales are generated by the integral operators in the form of

$$
\mathcal{L} u(x)=P . V . \int_{\mathbb{R}^{n}}\left\{u(x+y)-u(x)-(\nabla u(x) \cdot y) \chi_{B_{1}(0)}(y)\right\} d \mathfrak{m}(y),
$$

where a so-called Lévy measure $\mathfrak{m}$ satisfies

$$
\int_{\mathbb{R}^{n}} \min \left(1,|y|^{2}\right) d \mathfrak{m}(y)<+\infty .
$$

Since the operators are given in too much generality, we therefore restrict ourselves to considering only the operators given by symmetric kernels. In this case, the operator (1) can be written as

$$
\mathcal{L} u(x)=\int_{\mathbb{R}^{n}}\{u(x+y)+u(x-y)-2 u(x)\} K(y) d y,
$$

where a symmetric Lévy measure $m$ in 10 is given by a symmetric kernel $K(y)=K(-y)$. We note that the value of $\mathcal{L} u(x)$ is well-defined when $u$ is bounded in $\mathbb{R}^{n}$ and $C^{1,1}$ at $x$ (see Definition 2.1). Nonlinear integro-differential operators associated with the linear integrodifferential operators above arise naturally in the study of the stochastic control theory related to

$$
\mathcal{I} u(x)=\sup _{\alpha} \mathcal{L}_{\alpha} u(x),
$$

and game theory associated with

$$
\mathcal{I} u(x)=\inf _{\beta} \sup _{\alpha} \mathcal{L}_{\alpha \beta} u(x) .
$$

To study uniform regularity for such nonlinear integro-differential operators, the concept of ellipticity for integro-differential operators with respect to a class $\mathfrak{L}$ of the linear, integrodifferential operators (2) was introduced by Caffarelli and Silvestre [CS1]; see [CC] for elliptic second-order differential operators. In fact, the concept of ellipticity for integrodifferential operators $I$ is characterized by the following property:

$$
\inf _{\mathcal{L} \in \mathfrak{Q}} \mathcal{L} v(x) \leq \mathcal{I}[u+v](x)-\mathcal{I} u(x) \leq \sup _{\mathcal{L} \in \mathfrak{L}} \mathcal{L} v(x) .
$$

On the basis of this idea, the regularity theory for fully nonlinear elliptic integro-differential operators has been developed by using analytic techniques along the lines of the Krylov and Safonov [KS, CC] which dealt with elliptic second-order differential operators. We refer to [CS1, CS2] and references therein for uniform regularity results for symmetric integrodifferential operators of the fractional Laplacian type, where the regularity estimates do not blow up as the order $\sigma \in(0,2)$ of the operators tends to 2 . In the case when the kernels are nonsymmetric, the uniform regularity results can be found in [KL1, KL2, LD1]. We refer to [KL3, LD2] for results on the regularity of the parabolic integro-differetial operators.

In this paper, we establish the uniform regularity of viscosity solutions to fully nonlinear elliptic integro-differential equations associated with symmetric, regularly varying kernels at zero. We are mainly interested in the kernels $K$ for the integro-differential operator (2) satisfying

$$
\int_{\mathbb{R}^{n}} \min \left(1,|y|^{2}\right) K(y) d y<+\infty
$$

and

$$
(2-\sigma) \lambda \frac{l(|y|)}{|y|^{n}} \leq K(y) \leq(2-\sigma) \Lambda \frac{l(|y|)}{|y|^{n}}, \quad 0<\lambda \leq \Lambda<+\infty,
$$


where $l:(0,+\infty) \rightarrow(0,+\infty)$ is a locally bounded, regularly varying function at zero with index $-\sigma \in(-2,0)$; refer to Appendix $\mathrm{A}$ for regular variations. The kernels of the type (4) associated with regularly varying functions at zero appear in the study of the subordinate Brownian motions which are time changed Brownian motions by independent subordinators (i.e., nonnegative Lévy processes); see e.g. [KSV] for a potential theory of subordinate Brownian motions. There are known results on Harnack inequalities and Hölder estimates for integro-differential operators of this type with probabilistic proofs. In particular, Kassmann and Mimica $[\overline{\mathrm{KM}}]$ recently obtained Hölder type estimates for the linear integro-differential operators with regularly varying kernels at zero with index $-\sigma \in[-2,0]$ based on intrinsic scaling properties; the Hölder type estimates blow up as the order of the operator approaches 2. As an extension of the regularity results by Caffarelli and Silvestre [CS1], we obtain uniform regularity results of viscosity solutions for a certain class of fully nonlinear elliptic integro-differential operators associated with symmetric, regularly varying kernels at zero, which remain uniform as the order $\sigma \in(0,2)$ tends to 2 .

1.2. Integro-differential operators. As mentioned above, we study uniform regularity of viscosity solutions for a class of the fully nonlinear elliptic integro-differential operators associated with the kernels of the type:

$$
K(y) \asymp(2-\sigma) \frac{l(|y|)}{|y|^{n}}
$$

for a regularly varying function $l$ at zero with index $-\sigma \in(-2,0)$. For the purpose, we first summarize the properties of the regular variations that play an essential role in our analysis. In the entire article, a measurable function $l:(0,+\infty) \rightarrow(0,+\infty)$ which stays locally bounded away from 0 and $+\infty$, will be commonly assumed to satisfy the following properties.

Property 1.1. Let a measurable function $l:(0,1] \rightarrow(0,+\infty)$ be locally bounded away from 0 and $+\infty$. There exist positive constants $\sigma \in(0,2), a_{0} \geq 1$, and $\rho \in(0,1)$ satisfying the following.

(a) There exists $\delta \in\left[0, \frac{1}{2} \min (2-\sigma, \sigma)\right) \subset[0,1)$ such that

$$
\frac{l(s)}{l(r)} \leq a_{0} \max \left\{\left(\frac{s}{r}\right)^{-\sigma+\delta},\left(\frac{s}{r}\right)^{-\sigma-\delta}\right\} \quad \text { for } r, s \in(0,1] .
$$

(b) Define

$$
L(r):=\sigma \int_{r}^{1} \frac{l(s)}{s} d s
$$

Then we have for any $r \in(0, \rho)$

$$
\frac{1}{2} \leq \frac{L(r)}{l(r)} \leq 2
$$

(c) We assume that $l(1)=1$.

Influenced by Kassmann and Mimica $[\overline{\mathrm{KM}}]$, we introduce the monotone function $L$ above defined by using the given function $l$ in order to study scale invariant regularity estimates for the integro-differential operators associated with symmetric, regularly varying kernels at zero.

The function $l$ at infinity will be commonly assumed to satisfy the following property. 
Property 1.2. Let a measurable function $l:(0,+\infty) \rightarrow(0,+\infty)$ be locally bounded away from 0 and $+\infty$, and satisfy Property 1.1 There exists a positive constant $a_{\infty} \geq 1$ such that for some $\delta^{\prime} \in\left[0, \frac{1}{2} \min (2-\sigma, \sigma)\right) \subset[0,1)$

$$
\frac{l(s)}{l(r)} \leq a_{\infty} \max \left\{\left(\frac{s}{r}\right)^{-\sigma+\delta^{\prime}},\left(\frac{s}{r}\right)^{-\sigma-\delta^{\prime}}\right\} \quad \text { for } r, s \in[1,+\infty) .
$$

Typical examples of the functions satisfying Property 1.1 are regularly varying functions at zero with index $-\sigma \in(-2,0)$, and Property 1.2 is satisfied by assuming that the function $l$ varies regularly at infinity with index $-\sigma \in(-2,0)$; refer to Appendix $\AA$ for the definition of regular variations and their properties.

Example 1.3 (Regularly varying functions). (a) Trivial examples of such regularly varying functions at zero and infinity with index $-\sigma \in(-2,0)$ are

$$
(2-\sigma) r^{-\sigma} \text {. }
$$

The operator (2) with the choice above of the regularly varying function turns out to be the well-known fractional Laplacian operator $-(-\Delta)^{\sigma / 2}$ defined as

$$
-(-\Delta)^{\sigma / 2} u(x):=(2-\sigma) \int_{\mathbb{R}^{n}} \frac{u(x+y)+u(x-y)-2 u(x)}{|y|^{n+\sigma}} d y,
$$

which converges to the Laplacian operator as the order $\sigma \in(0,2)$ approaches 2 . We note that the factor $(2-\sigma)$ enables us to obtain second-order differential operators as the limits of integro-differential operators (see [DPV], CS1], for example) and hence uniform regularity results as the order $\sigma \in(0,2)$ goes to the classical one.

(b) Among nontrivial examples of regularly varying functions $l$ at zero with index $-\sigma$ are functions which are equal to the following functions near zero (see [BGT]):

$$
r^{-\sigma}\left(\log \frac{2}{r}\right)^{\beta}, r^{-\sigma}\left(\log \frac{2}{r^{2}}\right)^{\beta}, \quad \text { and } \quad r^{-\sigma}\left(\log \log \frac{2}{r}\right)^{\beta} \quad \text { for } \beta \in \mathbb{R} .
$$

(c) The following functions are non-logarithmic regularly varying functions $l$ at zero with index $-\sigma$ :

$$
r^{-\sigma} \exp \left(\left(\log \frac{2}{r}\right)^{\beta}\right) \quad \text { for } \beta \in(0,1)
$$

and

$$
r^{-\sigma} \exp \left(\log \frac{2}{r} \mid \log \log \frac{2}{r}\right) .
$$

For a certain class of regularly varying functions, the constants $a_{0} \geq 1, \rho \in(0,1)$ and $a_{\infty}$ in Properties 1.1 and 1.2 can be selected uniformly. Let $\sigma_{0} \in(0,2)$, and let a locally bounded function $l_{0}:(0,+\infty) \rightarrow(0,+\infty)$ be a slowly varying function at zero and infinity which varies regularly at zero and infinity with index 0 from the definition; see Appendix A. For $\sigma \in\left[\sigma_{0}, 2\right)$, define a regularly varying function $l_{\sigma}$ at zero and infinity with index $-\sigma \in\left(-2,-\sigma_{0}\right]$ by

$$
l_{\sigma}(r):=r^{-\sigma} l_{0}(r)^{2-\sigma}, \quad \forall r>0 .
$$

Making use of a theory of regular variations, we shall prove in Proposition 4.1 that the function $l_{\sigma}$ satisfies Properties 1.1 and 1.2 with uniform constants $a_{0}, a_{\infty} \geq 1$ and $\rho \in(0,1)$ for $\sigma \in\left[\sigma_{0}, 2\right)$, where the uniform constants depend only on dimension $n, \sigma_{0} \in(0,2)$, 
and a given slowly varying function $l_{0}$ at zero and infinity. Regarding the regularly varying functions of the type (5), we remark that for $\sigma \in(0,2)$ and $\beta \in(-\sigma, 2-\sigma)$, the kernel

$$
K(y) \asymp(2-\sigma)|y|^{-n-\sigma}\left(\log \frac{1}{|y|^{2}}\right)^{\beta / 2} \text { near zero }
$$

associated with the regularly varying function $l(r)=r^{-\sigma}\left(\log \frac{1}{r^{2}}\right)^{\beta / 2}$ describes the asymptotic behavior of the jumping kernel at zero of the subordinate process which has the characteristic exponent $\phi(s):=s^{\sigma}\left(1+\log s^{2}\right)^{\beta / 2}$; refer to a potential theory of subordinate Brownian motions [KSV].

To investigate a class of fully nonlinear elliptic integro-differential operators associated with symmetric, regularly varying kernels at zero, let $0<\lambda \leq \Lambda<+\infty$, and let a function $l:(0,+\infty) \rightarrow(0,+\infty)$ satisfy Properties 1.1 and 1.2 Owing to Properties 1.1 and 1.2 the following properties of the given function $l$ concerning the symmetric integro-differential operators are obtained; the proof can be found in Section 3

Lemma 1.4. Let a measurable function $l:(0,+\infty) \rightarrow(0,+\infty)$ be locally bounded away from 0 and $+\infty$, and satisfy Properties 1.1 and 1.2 with positive constants $\sigma \in(0,2), a_{0} \geq$ $1, a_{\infty} \geq 1$, and $\rho \in(0,1)$. Then we have the following:

(a)

$$
\frac{1}{2 a_{0}} \frac{r^{2} l(r)}{2-\sigma} \leq \int_{0}^{r} s l(s) d s \leq 2 a_{0} \frac{r^{2} l(r)}{2-\sigma}, \quad \forall r \in(0,1]
$$

$$
\sigma \int_{1}^{\infty} \frac{l(s)}{s} d s \leq 2 a_{\infty}
$$

Now, let $\mathfrak{L}(\lambda, \Lambda, l)$ denote the class of the following linear integro-differential operators with the kernels $K$ :

$$
\mathcal{L} u(x)=\int_{\mathbb{R}^{n}} \mu(u, x, y) K(y) d y,
$$

where $\mu(u, x, y):=u(x+y)+u(x-y)-2 u(x)$ and

$$
(2-\sigma) \lambda \frac{l(|y|)}{|y|^{n}} \leq K(y) \leq(2-\sigma) \Lambda \frac{l(|y|)}{|y|^{n}} .
$$

One can check that the kernels $K$ satisfying (6) with Properties 1.1 and 1.2 satisfy (3) by using Lemma 1.4. As mentioned in the introduction, we are concerned with the nonlinear integro-differential operator $\mathcal{I}$ in the form of

$$
\mathcal{I} u:=\inf _{\beta} \sup _{\alpha} \mathcal{L}_{\alpha \beta} u
$$

for some $\mathcal{L}_{\alpha \beta} \in \mathfrak{L}(\lambda, \Lambda, l)$. As extremal cases of such nonlinear integro-differential operators, the Pucci type extremal operators with respect to the class $\mathfrak{L}(\lambda, \Lambda, l)$ are defined as

$$
\begin{aligned}
& \mathcal{M}_{\mathfrak{L}(\lambda, \Lambda, l)}^{+} u:=\sup _{\mathcal{L} \in \mathfrak{Q}(\lambda, \Lambda, l)} \mathcal{L} u, \\
& \mathcal{M}_{\mathfrak{L}(\lambda, \Lambda, l)}^{-} u:=\inf _{\mathcal{L} \in \mathfrak{Q}(\lambda, \Lambda, l)} \mathcal{L} u .
\end{aligned}
$$

According to Lemma 2.6, the integro-differential operator $\mathcal{I}$ of the inf-sup type in (7) is elliptic with respect to $\mathfrak{L}(\lambda, \Lambda, l)$ in the nonlocal sense, which, in particular, implies

$$
\mathcal{M}_{\mathfrak{Q}(\lambda, \Lambda, l)}^{-} u \leq \mathcal{I} u \leq \mathcal{M}_{\mathfrak{Q}(\lambda, \Lambda, l)}^{+} u,
$$


where we refer to Definition2.3 for the nonlocal notion of the ellipticity. Thus we shall deal with a large class of the integro-differential operators defined in terms of the Pucci type extremal operators so as to establish uniform regularity estimates for the fully nonlinear elliptic integro-differential operators associated with symmetric, regularly varying kernels at zero.

1.3. Main results. Now we present our main results which extend the uniform regularity results of Caffarelli and Silvestre [CS1]. Below and hereafter, we denote $B_{R}:=B_{R}(0)$ for $R>0$.

Theorem 1.5 (Harnack inequality). Let $\sigma_{0} \in(0,2)$ and let a measurable function $l$ : $(0,+\infty) \rightarrow(0,+\infty)$ be locally bounded away from 0 and $+\infty$, and satisfy Properties 1.1 and 1.2 with the positive constants $\sigma \in\left[\sigma_{0}, 2\right), a_{0} \geq 1, a_{\infty} \geq 1$, and $\rho \in(0,1)$. For $0<R<1$, and $C_{0}>0$, let $u \in C\left(B_{2 R}\right)$ be a bounded, nonnegative function in $\mathbb{R}^{n}$ such that

$$
\mathcal{M}_{\mathfrak{Q}(\lambda, \Lambda, l)}^{-} u \leq C_{0} \quad \text { and } \quad \mathcal{M}_{\mathfrak{L}(\lambda, \Lambda, l)}^{+} u \geq-C_{0} \quad \text { in } B_{2 R}
$$

in the viscosity sense. Then there exist uniform constants $C>0$ and $\rho_{0} \in(0,1)$ such that

$$
\sup _{B_{R}} u \leq C\left(\inf _{B_{R}} u+\frac{C_{0}}{L\left(\rho_{0} R\right)}\right)
$$

where

$$
L(r):=\sigma \int_{r}^{1} \frac{l(s)}{s} d s \quad \forall 0<r<1,
$$

and $C>0$ and $\rho_{0} \in(0,1)$ are uniform constants depending only on $n, \lambda, \Lambda, \sigma_{0}, a_{0}, a_{\infty}$, and $\rho$.

Theorem 1.6 (Hölder estimate). Under the same assumption as in Theorem 1.5. let $u \in$ $C\left(B_{2 R}\right)$ be a bounded function in $\mathbb{R}^{n}$ such that

$$
\mathcal{M}_{\mathfrak{L}(\lambda, \Lambda, l)}^{-} u \leq C_{0} \quad \text { and } \quad \mathcal{M}_{\mathfrak{L}(\lambda, \Lambda, l)}^{+} u \geq-C_{0} \quad \text { in } B_{2 R}
$$

in the viscosity sense. Then we have

$$
R^{\alpha}[u]_{\alpha, B_{R}} \leq C\left(\|u\|_{L^{\infty}\left(\mathbb{R}^{n}\right)}+\frac{C_{0}}{L\left(\rho_{0} R\right)}\right),
$$

where $[u]_{\alpha, B_{R}}$ stands for the $\alpha$-Hölder seminorm on $B_{R}$, and the uniform constants $\alpha \in$ $(0,1), C>0$ and $\rho_{0} \in(0,1)$ depend only $n, \lambda, \Lambda, \sigma_{0}, a_{0}, a_{\infty}$, and $\rho$.

Remark 1.7. (i) According to Theorems 1.5 and 1.6, the Harnack inequality and Hölder estimate hold for viscosity solutions to the fully nonlinear elliptic integro-differential equations with respect to $\mathfrak{I}(\lambda, \Lambda, l)$. In fact, if $u$ is a viscosity solution to $\mathcal{I} u=f$ in $B_{2 R}$ for an elliptic integro-differential operator with respect to $\mathfrak{L}(\lambda, \Lambda, l)$ and $f \in L^{\infty}\left(B_{2 R}\right)$, then $u$ satisfies

$$
\mathcal{M}_{\mathfrak{L}(\lambda, \Lambda, l)}^{-} u \leq\|f\|_{L^{\infty}\left(B_{2 R}\right)}+|\mathcal{I} 0|, \quad \text { and } \quad \mathcal{M}_{\mathfrak{L}(\lambda, \Lambda, l)}^{+} u \geq-\|f\|_{L^{\infty}\left(B_{2 R}\right)}-|\mathcal{I} 0|
$$

in the viscosity sense. Thus, applying Theorems 1.5 and 1.6 , the regularity results follow.

(ii) For any regularly varying function $l$ at zero and infinity with index $-\sigma \in(-2,0)$ which stays away from 0 and $+\infty$, we obtain the Harnack inequality and Hölder estimate for the elliptic integro-differential operators with respect to $\mathfrak{L}(\lambda, \Lambda, l)$ as a corollary. Here, the constants in the regularity estimates above depend only on $n, \lambda, \Lambda$, and the given regularly varying function $l$. 
Making use of Theorem 1.6 we establish the $C^{1, \alpha}$ estimate for the fully nonlinear elliptic integro-differential operators associated with regularly varying kernels at zero and infinity provided that the kernels satisfy a cancelation property at infinity; see Subsection 3.6 Furthermore, the Hölder estimate for the elliptic integro-differential operators associated with truncated kernels at infinity is also obtained in Subsection 3.7, which is important for applications. In fact, the assumption of the kernels at infinity for the Hölder estimate in Theorem 1.6 can be weakened replacing Property 1.2 by the boundedness of the integral at infinity

$$
(2-\sigma) \int_{1}^{\infty} \frac{l(s)}{s} d s \leq a_{\infty}
$$

for some $a_{\infty}>0$; in the following, we rephrase Theorem 3.14 by assuming 97 .

Theorem 1.8. Let $\sigma_{0} \in(0,2)$ and let $l:(0,+\infty) \rightarrow[0,+\infty)$ be a measurable function which is locally bounded away from 0 and $+\infty$ on $(0,1]$, and satisfy Property 1.1 with the positive constants $\sigma \in\left[\sigma_{0}, 2\right), a_{0} \geq 1$, and $\rho \in(0,1)$. We assume that

$$
(2-\sigma) \int_{1}^{\infty} \frac{l(s)}{s} d s \leq a_{\infty} \text { for some } a_{\infty}>0 .
$$

For $0<R<1$, and $C_{0}>0$, let $u \in C\left(B_{2 R}\right)$ be a bounded function in $\mathbb{R}^{n}$ such that

$$
\mathcal{M}_{\mathfrak{Q}(\lambda, \Lambda, l)}^{-} u \leq C_{0} \text { and } \mathcal{M}_{\mathfrak{Q}(\lambda, \Lambda, l)}^{+} u \geq-C_{0} \text { in } B_{2 R}
$$

in the viscosity sense. Then we have

$$
R^{\alpha}[u]_{\alpha, B_{R}} \leq C\left(\|u\|_{L^{\infty}\left(\mathbb{R}^{n}\right)}+\frac{C_{0}}{L\left(\rho_{0} R\right)}\right),
$$

where the uniform constants $\alpha \in(0,1), C>0$ and $\rho_{0} \in(0,1)$ depend only $n, \lambda, \Lambda, \sigma_{0}, a_{0}, \rho$ and $a_{\infty}$.

With the help of Proposition 4.1, Theorems 1.5, 1.6 and 1.8 yield the uniform Harnack inequality and Hölder estimate for the fully nonlinear elliptic integro-differential operators with respect to the class $\mathfrak{L}\left(\lambda, \Lambda, l_{\sigma}\right)$ associated with symmetric, regularly varying kernels of the type (5) for $\sigma \in\left[\sigma_{0}, 2\right) \subset(0,2)$, where the regularity estimates do not blow up as the order $\sigma$ goes to 2 .

Theorem 1.9 (Uniform estimates for the operators associated with the kernels of the type (5)). Let $\sigma_{0} \in(0,2)$, and let a measurable function $l_{0}:(0,+\infty) \rightarrow(0,+\infty)$ be locally bounded away from 0 and $+\infty$, and vary slowly at zero and infinity such that $l_{0}(1)=1$. For $\sigma \in\left[\sigma_{0}, 2\right)$, define

$$
\begin{aligned}
l_{\sigma}(r) & :=r^{-\sigma} l_{0}(r)^{2-\sigma}, \quad \forall r \in(0,+\infty), \\
L_{\sigma}(r) & :=\sigma \int_{r}^{1} s^{-1-\sigma} l_{0}(s)^{2-\sigma} d s, \quad \forall r \in(0,1] .
\end{aligned}
$$

(a) For $0<R<1$, and $C_{0}>0$, let $u \in C\left(B_{2 R}\right)$ be a bounded, nonnegative function in $\mathbb{R}^{n}$ such that

$$
\mathcal{M}_{\mathfrak{Q}\left(\lambda, \Lambda, l_{\sigma}\right)}^{-} u \leq C_{0} \quad \text { and } \quad \mathcal{M}_{\mathfrak{Q}\left(\lambda, \Lambda, l_{\sigma}\right)}^{+} u \geq-C_{0} \quad \text { in } B_{2 R}
$$

in the viscosity sense. Then we have

$$
\sup _{B_{R}} u \leq C\left(\inf _{B_{R}} u+\frac{C_{0}}{L_{\sigma}\left(\rho_{0} R\right)}\right) .
$$


(b) Let $u \in C\left(B_{2 R}\right)$ be a bounded function in $\mathbb{R}^{n}$ such that

$$
\mathcal{M}_{\mathfrak{Q}\left(\lambda, \Lambda, l_{\sigma}\right)}^{-} u \leq C_{0} \quad \text { and } \quad \mathcal{M}_{\mathfrak{Q}\left(\lambda, \Lambda, l_{\sigma}\right)}^{+} u \geq-C_{0} \quad \text { in } B_{2 R}
$$

in the viscosity sense. Then we have

$$
R^{\alpha}[u]_{\alpha, B_{R}} \leq C\left(\|u\|_{L^{\infty}\left(\mathbb{R}^{n}\right)}+\frac{C_{0}}{L_{\sigma}\left(\rho_{0} R\right)}\right),
$$

where $C>0, \rho_{0} \in(0,1)$ and $\alpha \in(0,1)$ are uniform constants depending only on $n, \lambda, \Lambda, \sigma_{0}$ and the slowly varying function $l_{0}$ at zero and infinity.

In Theorem 1.9, we establish the uniform Harnack inequality and Hölder estimate for a class of fully nonlinear elliptic integro-differential operators associated with the kernels $K_{\sigma}$ in the form of

$$
(2-\sigma) \lambda \frac{l_{0}(|y|)^{2-\sigma}}{|y|^{n+\sigma}} \leq K_{\sigma}(y) \leq(2-\sigma) \Lambda \frac{l_{0}(|y|)^{2-\sigma}}{|y|^{n+\sigma}} .
$$

In the case when $l_{0} \equiv 1$, we observe that for $\sigma \in\left[\sigma_{0}, 2\right)$,

$$
\begin{aligned}
l_{\sigma}(r) & =r^{-\sigma} \\
L_{\sigma}(r) & =r^{-\sigma}-1 \geq\left(1-2^{-\sigma_{0}}\right) r^{-\sigma}, \quad \forall r \in(0,1 / 2) .
\end{aligned}
$$

This implies that our results recover [CS1, Theorem 11.1, Theorem 12.1]. In particular, considering the following example of slowly varying functions at zero: for $l_{0}(r):=$ $\left(\log \frac{2}{r^{2}}\right)$,

$$
l_{0}^{\beta}(r)=\left(\log \frac{2}{r^{2}}\right)^{\beta} \quad \forall r \in(0,1), \beta \in \mathbb{R},
$$

Theorem 1.9 asserts the uniform Harnack inequality and Hölder estimate of the elliptic integro-differential operators associated with the regularly varying kernel $K_{\sigma, \beta}$ at zero with index $-\sigma \in\left(-2,-\sigma_{0}\right]$ for $\beta \in \mathbb{R}$ :

$$
(2-\sigma) \frac{\lambda}{|y|^{n+\sigma}}\left(\log \frac{2}{|y|^{2}}\right)^{\beta(2-\sigma)} \leq K_{\sigma, \beta}(y) \leq(2-\sigma) \frac{\Lambda}{|y|^{n+\sigma}}\left(\log \frac{2}{|y|^{2}}\right)^{\beta(2-\sigma)} \quad \text { near zero, }
$$

where the uniform constants in the regularity estimates depend only on $n, \lambda, \Lambda, \sigma_{0}, \beta$ and the given slowly varying function $l_{0}$ at zero.

Lastly, we have the following theorem as a corollary of Theorem 1.8 by imposing (9) instead of Property 1.2

Theorem 1.10. Let $\sigma_{0} \in(0,2)$, and let a measurable function $l_{0}:(0,+\infty) \rightarrow[0,+\infty)$ be locally bounded away from 0 and $+\infty$ on $(0,1]$, and vary slowly at zero such that $l_{0}(0)=1$. For $\sigma \in\left[\sigma_{0}, 2\right)$, define $l_{\sigma}:(0,+\infty) \rightarrow[0,+\infty)$ and $L_{\sigma}:(0,1] \rightarrow(0,+\infty)$ as $[10$. We assume that

$$
(2-\sigma) \int_{1}^{\infty} \frac{l_{\sigma}(s)}{s} d s \leq a_{\infty} \quad \text { for some } a_{\infty}>0 .
$$

For $0<R<1$, and $C_{0}>0$, let $u \in C\left(B_{2 R}\right)$ be a bounded function in $\mathbb{R}^{n}$ such that

$$
\mathcal{M}_{\mathfrak{Q}(\lambda, \Lambda, l)}^{-} u \leq C_{0} \quad \text { and } \quad \mathcal{M}_{\mathfrak{Q}(\lambda, \Lambda, l)}^{+} u \geq-C_{0} \quad \text { in } B_{2 R}
$$

in the viscosity sense. Then we have

$$
R^{\alpha}[u]_{\alpha, B_{R}} \leq C\left(\|u\|_{L^{\infty}\left(\mathbb{R}^{n}\right)}+\frac{C_{0}}{L\left(\rho_{0} R\right)}\right),
$$

where the uniform constants $\alpha \in(0,1), C>0$ and $\rho_{0} \in(0,1)$ depend only $n, \lambda, \Lambda, \sigma_{0}, a_{\infty}$, and the slowly varying function $l_{0}$ at zero. 
The rest of the paper is organized as follows. Section 2 contains an introduction to viscosity solutions, the ellipticity for integro-differential operators and their properties. Section 3 is devoted to the proof of the uniform regularity estimates for a class of fully nonlinear elliptic integro-differential operators associated with the kernels satisfying (6) with Properties 1.1 and 1.2. In Section 4, we prove Proposition 4.1 and obtain Theorems 1.9 and 1.10 from Theorems 1.5, 1.6 and 1.8. In Appendix A we give the definitions of regularly and slowly varying functions and summarize their important properties which are used in the paper.

\section{Viscosity SOLUTIONS}

In this section, we give an introduction to the notions of viscosity solutions and the ellipticity for integro-differential operators as in [CS1]; see also [LD1, KL2]. Important properties of viscosity solutions such as the stabilities under uniform convergence and the comparison principle are also provided; refer to [CC] for the local case. We begin with the concept of $C^{1,1}$ at the point.

Definition 2.1 ( $C^{1,1}$ at the point). Let $x \in \mathbb{R}^{n}$. A function $\varphi$ is said to be $C^{1,1}$ at the point $x$, denoted by $\varphi \in C^{1,1}(x)$, if there exist a vector $p \in \mathbb{R}^{n}$ and a number $M>0$ such that

$$
|\varphi(x+y)-\varphi(x)-p \cdot y| \leq M|y|^{2} \quad \text { for small } y \in \mathbb{R}^{n} .
$$

For a set $\Omega \subset \mathbb{R}^{n}$, we say that $\varphi$ is $C^{1,1}$ in $\Omega$ when (11) holds for any $x \in \Omega$ with a uniform constant $M>0$.

Now, we recall the viscosity solutions for integro-differential operators.

Definition 2.2 (Viscosity solution). Let $\Omega \subset \mathbb{R}^{n}$ be an open set and let $f$ be a function in $\Omega$. A bounded function $u: \mathbb{R}^{n} \rightarrow \mathbb{R}$ which is upper (lower) semi-continuous in $\bar{\Omega}$ is called a viscosity subsolution (supersolution) to the integro-differential equation $\mathcal{I} u=f$ in $\Omega$ and we write $\mathcal{I} u \geq f$ in $\Omega\left(\mathcal{I} u \leq f\right.$ in $\Omega$ ) when the following holds: if a $C^{2}$-function $\varphi$ touches $u$ from above (below) at $x \in \Omega$ in a small neighborhood $N$ of $x$, i.e.,

(i) $\varphi(x)=u(x)$,

(ii) $\phi>u(\phi<u)$ in $N \backslash\{x\}$,

then the function $v$ defined as

$$
v:= \begin{cases}\varphi & \text { in } N, \\ u & \text { in } \mathbb{R}^{n} \backslash N,\end{cases}
$$

satisfies $\mathcal{I} v(x) \geq f(x)(\mathcal{I} v(x) \leq f(x))$. We say $u$ is a viscosity solution if $u$ is both a viscosity subsolution and $a$ viscosity supersolution.

Here, we consider bounded viscosity solutions for nonlocal operators for simplicity. Our method to prove the Harnack inequality for viscosity solutions can be also employed under the assumption that the viscosity solutions have a certain growth rate at infinity related to a class of integro-differential operators to deal with; see [BI] for viscosity solutions to integro-differential equations in a general framework.

The notion of ellipticity for integro-differential operators is defined making use of a nonlocal version of the Pucci extremal operators. For given $0<\lambda \leq \Lambda<+\infty$, and a function $l:(0,+\infty) \rightarrow[0,+\infty)$ satisfying Property 1.1] and (9), which stays away from 0 and $+\infty$ on $(0,1]$, let $\mathfrak{L}(\lambda, \Lambda, l)$ denote the class of the following linear integro-differential operators with the kernels $K$ satisfying (6):

$$
\mathcal{L} u(x)=\int_{\mathbb{R}^{n}} \mu(u, x, y) K(y) d y,
$$


where $\mu(u, x, y):=u(x+y)+u(x-y)-2 u(x)$. We recall the Pucci type extremal operators:

$$
\mathcal{M}_{\mathfrak{Q}(\lambda, \Lambda, l)}^{+} u:=\sup _{\mathcal{L} \in \mathfrak{Q}(\lambda, \Lambda, l)} \mathcal{L} u, \quad \text { and } \mathcal{M}_{\mathfrak{L}(\lambda, \Lambda, l)}^{-} u:=\inf _{\mathcal{L} \in \mathfrak{Q}(\lambda, \Lambda, l)} \mathcal{L} u \text {. }
$$

One can check that

$$
\begin{aligned}
& \mathcal{M}_{\mathfrak{Q}(\lambda, \Lambda, l)}^{+} u(x)=(2-\sigma) \int_{\mathbb{R}^{n}}\left\{\Lambda \mu^{+}(u, x, y)-\lambda \mu^{-}(u, x, y)\right\} \frac{l(|y|)}{|y|^{n}} d y, \\
& \mathcal{M}_{\mathfrak{Q}(\lambda, \Lambda, l)}^{-} u(x)=(2-\sigma) \int_{\mathbb{R}^{n}}\left\{\lambda \mu^{+}(u, x, y)^{+}-\Lambda \mu^{-}(u, x, y)\right\} \frac{l|y|)}{|y|^{n}} d y,
\end{aligned}
$$

where $\mu^{ \pm}(u, x, y):=\max \{ \pm \mu(u, x, y), 0\}$.

In terms of the Pucci type operators with respect to the class $\mathfrak{I}(\lambda, \Lambda, l)$, the elliptic integro-differential operators with respect to $\mathfrak{L}(\lambda, \Lambda, l)$ are defined as below, which we can apply our results to.

Definition 2.3 (Ellipticity for nonlocal operators). An operator $\mathcal{I}$ is said to be elliptic with respect to the class $\mathfrak{Q}(\lambda, \Lambda, l)$ if it satisfies the following.

(i) If a bounded function $u$ in $\mathbb{R}^{n}$ is of $C^{1,1}(x)$, then $I u(x)$ is defined classically.

(ii) If a bounded function $u$ in $\mathbb{R}^{n}$ is of $C^{1,1}(\Omega)$ for an open set $\Omega$, then $I u(x)$ is continuous in $\Omega$.

(iii) For bounded functions $u \in C^{1,1}(x)$, and $v \in C^{1,1}(x)$, we have

$$
\mathcal{M}_{\mathfrak{Q}(\lambda, \Lambda, l)}^{-} v(x) \leq \mathcal{I}[u+v](x)-\mathcal{I} u(x) \leq \mathcal{M}_{\mathfrak{Q}(\lambda, \Lambda, l)}^{+} v(x) .
$$

Remark 2.4. If a bounded function $u$ in $\mathbb{R}^{n}$ is $C^{1,1}$ at the point $x$, then the Pucci operators $\mathcal{M}_{\mathfrak{Q}(\lambda, \Lambda, l)}^{ \pm} u(x)$ are defined classically due to the properties (a) and (b) in Lemma1.4.

In Definition 2.2. a $C^{2}$-test function $\varphi$ can be taken to be $C^{1,1}$ only at the contact point $x$ for elliptic integro-differential operators. We are led to consider a larger set of test functions and a stronger concept of the viscosity solution, however, those approaches turn out to be equivalent thanks to the following lemma. The proof is similar to one of [CS1, Lemma 4.3] with the help of Lemma 1.4, see also [LD1, Lemma 4.3].

Lemma 2.5. Let $\Omega \subset \mathbb{R}^{n}$ be an open set and let $I$ be an elliptic integro-differential operator with respect to $\mathfrak{I}=\mathfrak{Q}(\lambda, \Lambda, l)$. Let $u: \mathbb{R}^{n} \rightarrow \mathbb{R}$ satisfy $\mathcal{I} u \geq f$ in $\Omega$ in the viscosity sense. We assume that a function $\varphi \in C^{1,1}(x)$ (for a point $x \in \Omega$ ) touches $u$ from above at $x$ in a small neighborhood $N$ of $x$. Then the function $v$ defined as

$$
v:= \begin{cases}\varphi & \text { in } N \\ u & \text { in } \mathbb{R}^{n} \backslash N\end{cases}
$$

satisfies $\mathcal{I} v(x) \geq f(x)$ in the classical sense.

Due to Property 1.1] and (9) (Lemma 1.4], the results of [CS1] on viscosity solutions for the elliptic integro-differential operators hold true for our elliptic integro-differential operators with respect to the class $\mathfrak{L}(\lambda, \Lambda, l)$. First, the following lemma concerns the nonlinear integro-differential operators of the inf-sup type (7); the proofs can be found in [CS1, Sections 3 and 4].

Lemma 2.6 (Properties of the inf-sup type operators). Let $\mathcal{I}$ be the operator in the form of (7). Then we have the following.

(a) I is an elliptic integro-differential operator with respect to $\mathfrak{Q}(\lambda, \Lambda, l)$, that is, $\mathcal{I}$ satisfies: 
(i) For bounded functions $u$ and $v$ which are $C^{1,1}$ at $x$,

$$
\mathcal{M}_{\mathfrak{Q}(\lambda, \Lambda, l)}^{-} v(x) \leq \mathcal{I}[u+v](x)-\mathcal{I} u(x) \leq \mathcal{M}_{\mathfrak{Q}(\lambda, \Lambda, l)}^{+} v(x) .
$$

(ii) If a bounded function $u$ in $\mathbb{R}^{n}$ is $C^{1,1}$ in an open set $\Omega$, then $\mathcal{I} u(x)$ is continuous in $\Omega$.

(b) If $u$ is a viscosity subsolution to $I u=f$ in an open set $\Omega$, and a function $\varphi \in C^{1,1}(x)$ (for $x \in \Omega$ ) touches $u$ from above at $x$ in a small neighborhood of $x$, then $I u(x)$ is defined classically and $\mathcal{I} u(x) \geq f(x)$.

The viscosity solutions to the elliptic integro-differential equations have nice stability properties with respect to uniform convergence. Recalling the definition of $\Gamma$-convergence, a slightly stronger stability of viscosity solutions under $\Gamma$-convergence in Lemma 2.8 is quoted from [CS1, Lemma 4.5].

Definition 2.7 ( $\Gamma$-convergence). We say a sequence of lower semi-continuous functions $u_{k}$ $\Gamma$-converges to $u$ in a set $\Omega \subset \mathbb{R}^{n}$ if it satisfies the following conditions:

(i) For every sequence $x_{k} \rightarrow x$ in $\Omega$,

$$
\liminf _{k \rightarrow \infty} u_{k}\left(x_{k}\right) \geq u(x)
$$

(ii) For every $x \in \Omega$, there exists a sequence $x_{k} \rightarrow x$ in $\Omega$ such that

$$
\limsup _{k \rightarrow \infty} u_{k}\left(x_{k}\right)=u(x) \text {. }
$$

Lemma 2.8 (Stability). Let $\mathcal{I}$ be an elliptic operator with respect to the class $\mathcal{L}(\lambda, \Lambda, l)$. For an open set $\Omega \subset \mathbb{R}^{n}$, let $u_{k}$ be a sequence of functions that are uniformly bounded in $\mathbb{R}^{n}$ such that

(i) $I u_{k} \leq f_{k}$ in $\Omega$ in the viscosity sense,

(ii) $u_{k} \rightarrow u$ in the $\Gamma$-sense in $\Omega$,

(iii) $u_{k} \rightarrow$ u a.e. in $\mathbb{R}^{n}$,

(iv) $f_{k} \rightarrow f$ locally uniformly in $\Omega$ for some continuous function $f$.

Then $I u \leq f$ in $\Omega$ in the viscosity sense.

As a corollary, we obtain the stability property under uniform convergence.

Corollary 2.9. Let $\mathcal{I}$ be an elliptic operator with respect to the class $\mathfrak{L}(\lambda, \Lambda, l)$. For an open set $\Omega \subset \mathbb{R}^{n}$, let $u_{k} \in C(\Omega)$ be a sequence of functions that are uniformly bounded in $\mathbb{R}^{n}$ such that

(i) $\mathcal{I} u_{k}=f_{k}$ in $\Omega$ in the viscosity sense,

(ii) $u_{k} \rightarrow$ u locally uniformly in $\Omega$,

(iii) $u_{k} \rightarrow$ u a.e. in $\mathbb{R}^{n}$,

(iv) $f_{k} \rightarrow f$ locally uniformly in $\Omega$ for some continuous function $f$.

Then $\mathcal{I} u=f$ in $\Omega$ in the viscosity sense.

Lemma 2.10 quoted from [CS1, Theorem 5.9] states that the difference of two viscosity solutions solves an equation in the same ellipticity class. In the proof, Jensen's approach [J] using the inf- and sup-convolutions was employed to compare two viscosity solutions to the fully nonlinear elliptic integro-differential equations (see also $[\mathrm{A}]$ ); we refer to [CC, Chapter 5] for the local case.

Lemma 2.10. Let $\mathcal{I}$ be an elliptic operator with respect to the class $\mathfrak{L}(\lambda, \Lambda, l)$. For an open set $\Omega \subset \mathbb{R}^{n}$, let $u$ and $v$ be bounded in $\mathbb{R}^{n}$ such that

$$
\mathcal{I} u \geq f, \quad \text { and } \quad \mathcal{I} v \leq g \text { in } \Omega
$$


in the viscosity sense for two continuous functions $f$ and $g$. Then

$$
\mathcal{M}_{\mathfrak{Q}(\lambda, \Lambda, l)}^{+}(u-v) \geq f-g \text { in } \Omega
$$

in the viscosity sense.

The comparison principle for the elliptic integro-differential operators as in [CS1, Theorem 5.2] follows from Lemma 2.10 with the help of a barrier function given in Lemma 2.11, see also [CS1, Assumption 5.1 and Lemma 5.10].

Lemma 2.11. For a given $R \geq 1$, there exists $\delta_{R}>0$ such that the function $\varphi_{R}(x):=$ $\min \left(1, \frac{|x|^{2}}{4 R^{2}}\right)$ satisfies

$$
\mathcal{M}_{\mathcal{Q}(\lambda, \Lambda, l)}^{-} \varphi_{R} \geq \delta_{R} \quad \text { in } B_{R}
$$

Proof. Let $x \in B_{R}$. If $x \pm y \in B_{2 R}$, then we have $\mu\left(\varphi_{R}, x, y\right)=\frac{|y|^{2}}{2 R^{2}}$. If $x+y \notin B_{2 R}$, then $\mu\left(\varphi_{R}, x, y\right) \geq 1-\frac{|x|^{2}}{2 R^{2}} \geq \frac{1}{2}$. Thus it follows that for $x \in B_{R}$,

$$
\mathcal{M}^{-} \varphi_{R}(x)=(2-\sigma) \lambda \int_{\mathbb{R}^{n}} \mu\left(\varphi_{R}, x, y\right) \frac{l(|y|)}{|y|^{n}} d y \geq(2-\sigma) \lambda \int_{B_{R}} \frac{|y|^{2}}{2 R^{2}} \frac{l(|y|)}{|y|^{n}} d y=: \delta_{R}>0 .
$$

Lastly, we state the comparison principle for the fully nonlinear elliptic integro-differential operators with respect to $\mathfrak{L}(\lambda, \Lambda, l)$; the proof is the same as one for Theorem 5.2 of [CS1].

Theorem 2.12 (Comparison principle). Let I be an elliptic operator with respect to the class $\mathfrak{Q}(\lambda, \Lambda, l)$. For a bounded open set $\Omega \subset \mathbb{R}^{n}$, let $u$ and $v$ be bounded in $\mathbb{R}^{n}$ such that

(i) $\mathcal{I} u \geq f$ and $\mathcal{I} v \leq f$ in $\Omega$ in the viscosity sense for some continuous functions $f$,

(ii) $u \leq v$ in $\mathbb{R}^{n} \backslash \Omega$.

Then $u \leq v$ in $\Omega$.

\section{REGULARITY ESTIMATES FOR INTEGRO-DIFFERENTIAL OPERATORS WITH REGULARLY VARYING} KERNELS

This section is mainly devoted to proving Theorems 1.5 and 1.6 , which will provide the Harnack inequality and Hölder estimate for fully nonlinear elliptic integro-differential operators associated with symmetric, regularly varying kernel at zero and infinity as mentioned in Remark 1.7 Throughout this section, let $0<\lambda \leq \Lambda<+\infty$, and let a measurable function $l:(0,+\infty) \rightarrow(0,+\infty)$ be locally bounded away from 0 and $+\infty$, and satisfy Properties 1.1 and 1.2 with the positive constants $\sigma \in\left[\sigma_{0}, 2\right), a_{0} \geq 1, a_{\infty} \geq 1$, and $\rho \in(0,1)$ for a given $\sigma_{0} \in(0,2)$. As in Subsection 1.2, let $\mathfrak{L}(\lambda, \Lambda, l)$ be the class of all linear integrodifferential operators

$$
\mathcal{L} u(x)=\int_{\mathbb{R}^{n}} \mu(u, x, y) K(y) d y
$$

with the kernels $K$ satisfying

$$
(2-\sigma) \lambda \frac{l(|y|)}{|y|^{n}} \leq K(y) \leq(2-\sigma) \Lambda \frac{l(|y|)}{|y|^{n}},
$$

where $\mu(u, x, y):=u(x+y)+u(x-y)-2 u(x)$. In order to prove the uniform regularity estimates for a class of viscosity solutions to the elliptic integro-differential equations with respect to the class $\mathfrak{L}(\lambda, \Lambda, l)$, we will deal with the Pucci type extremal operators $\mathcal{M}_{\mathfrak{Q}(\lambda, \Lambda, l)}^{ \pm}$, defined as $(8)$ and simply denoted by $\mathcal{M}^{ \pm}$, since the elliptic operator $\mathcal{I}$ satisfies that

$$
\mathcal{M}_{\mathfrak{Q}(\lambda, \Lambda, l)}^{-} \leq \mathcal{I}-\mathcal{I}[0] \leq \mathcal{M}_{\mathfrak{Q}(\lambda, \Lambda, l)}^{+} .
$$


Before we proceed to regularity estimates for viscosity solutions to nonlocal equations, we study the important properties of the given function $l$ satisfying Properties 1.1 and 1.2 which will be used later.

Lemma 3.1. Let a measurable function $l:(0,+\infty) \rightarrow(0,+\infty)$ be locally bounded away from 0 and $+\infty$, and satisfy Properties 1.1 and 1.2 with positive constants $\sigma \in(0,2)$, $a_{0} \geq 1, a_{\infty} \geq 1$, and $\rho \in(0,1)$. Then we have the following.

(a) For $r \in(0,1]$,

$$
\frac{1}{2 a_{0}} \frac{r^{2} l(r)}{2-\sigma} \leq \int_{0}^{r} s l(s) d s \leq 2 a_{0} \frac{r^{2} l(r)}{2-\sigma} .
$$

(b) For $r \in(0,1]$,

$$
\int_{0}^{r} s^{3} l(s) d s \leq a_{0} r^{4} l(r)
$$

(c) For $r \in(0,1]$,

$$
L(r):=\sigma \int_{r}^{1} \frac{l(s)}{s} d s \geq \frac{1}{2 a_{0}^{2}}\left(r^{-\sigma / 2}-1\right) .
$$

In particular, for $\sigma \in\left[\sigma_{0}, 2\right)$, we have

$$
\frac{1}{2 a_{0}^{2}}\left(r^{-\sigma_{0} / 2}-1\right) \leq L(r) \leq 2 a_{0}^{2} r^{-2}, \quad \forall r \in(0,1] .
$$

(d)

$$
\sigma \int_{1}^{\infty} \frac{l(s)}{s} d s \leq 2 a_{\infty} .
$$

Proof. Using Property 1.1, we have that for $r \in(0,1]$,

$$
\begin{aligned}
\int_{0}^{r} s l(s) d s & =l(r) \int_{0}^{r} s \frac{l(s)}{l(r)} d s \leq a_{0} r^{\sigma+\delta} l(r) \int_{0}^{r} s^{1-\sigma-\delta} d s \\
& \leq a_{0} r^{\sigma+\delta} l(r) \frac{1}{2-\sigma-\delta} r^{2-\sigma-\delta} \leq \frac{2 a_{0}}{2-\sigma} r^{2} l(r)
\end{aligned}
$$

since $\delta \in\left[0, \frac{1}{2} \min (2-\sigma, \sigma)\right)$. In a similar way, we deduce that for $r \in(0,1]$

$$
\frac{1}{2 a_{0}} \frac{r^{2} l(r)}{2-\sigma} \leq \int_{0}^{r} \operatorname{sl}(s) d s \leq 2 a_{0} \frac{r^{2} l(r)}{2-\sigma}
$$

and

$$
\int_{0}^{r} s^{3} l(s) d s \leq a_{0} r^{4} l(r)
$$

Recalling from Property 1.1 that for $r \in(0,1]$

$$
L(r):=\sigma \int_{r}^{1} \frac{l(s)}{s} d s,
$$


it follows that

$$
\begin{aligned}
L(r) & =\sigma l(r) \int_{r}^{1} \frac{1}{s} \frac{l(s)}{l(r)} d s \\
& \geq \frac{\sigma}{a_{0}} r^{\sigma+\delta} l(r) \int_{r}^{1} s^{-1-\sigma-\delta} d s=\frac{\sigma}{a_{0}} r^{\sigma+\delta} l(r) \frac{1}{\sigma+\delta}\left(r^{-\sigma-\delta}-1\right) \\
& \geq \frac{1}{2 a_{0}} r^{\sigma+\delta} \frac{l(1)}{a_{0}} r^{-\sigma+\delta}\left(r^{-\sigma-\delta}-1\right)=\frac{1}{2 a_{0}^{2}}\left(r^{-\sigma+\delta}-r^{2 \delta}\right) \\
& \geq \frac{1}{2 a_{0}^{2}}\left(r^{-\sigma / 2}-1\right) .
\end{aligned}
$$

Arguing in a similar way,, we have

$$
\frac{1}{2 a_{0}^{2}}\left(r^{-\sigma / 2}-1\right) \leq L(r) \leq 2 a_{0}^{2} r^{-2}, \quad \forall r \in(0,1] .
$$

Since $l(1)=1$, Property 1.2 yields that

$$
\sigma \int_{1}^{\infty} \frac{l(s)}{s} d s \leq \sigma a_{\infty} \int_{1}^{\infty} s^{-1-\sigma+\delta^{\prime}} d s \leq a_{\infty} \frac{\sigma}{\sigma-\delta^{\prime}} \leq 2 a_{\infty},
$$

completing the proof.

3.1. Aleksandrov-Bakelman-Pucci type estimate. First, we extend the nonlocal AleksandrovBakelman-Pucci(ABP) estimate by Caffarelli and Silvestre [CS1, Lemma 8.1] for fully nonlinear elliptic integro-differential operators with respect to $\mathfrak{L}(\lambda, \Lambda, l)$.

Lemma 3.2. Let $R \in(0,1)$ and $\rho_{0} \in(0,1)$. Let $r_{k}:=\rho_{0} 2^{-\frac{1}{2(2-\sigma)}-k} R$, and $\mathcal{R}_{k}(x):=B_{r_{k}}(x) \backslash$ $B_{r_{k+1}}(x)$ for $k \in \mathbb{N} \cup\{0\}$ and $x \in \mathbb{R}^{n}$. Let $u$ be a viscosity subsolution of

$$
\mathcal{M}_{\mathfrak{Q}(\lambda, \Lambda, l)}^{+} u=-f \quad \text { on } B_{R}
$$

such that $u \leq 0$ in $\mathbb{R}^{n} \backslash B_{R}$, and let $\Gamma$ be the concave envelope of $u^{+}:=\max \{u, 0\}$ in $B_{3 R}$. Then there exists a uniform constant $\tilde{C}:=\frac{c_{n} a_{0}}{\lambda \rho_{0}^{4}} \sup _{\sigma \in\left[\sigma_{0}, 2\right)}\left(\frac{1-2^{-2(2-\sigma)}}{2-\sigma}\right)>0$ such that for each $x \in\{u=\Gamma\}$ and $M>0$, we find $k \in \mathbb{N} \cup\{0\}$ satisfying

$$
\left|\left\{y \in \mathcal{R}_{k}(x): u(y)<u(x)+(y-x) \cdot \nabla \Gamma(x)-M r_{k}^{2}\right\}\right| \leq \frac{\tilde{C}}{l(R) R^{2}} \frac{f(x)}{M}\left|\mathcal{R}_{k}(x)\right|,
$$

where $\nabla \Gamma(x)$ stands for an element of the superdifferential of $\Gamma$ at $x$, and $c_{n}>0$ depends only on dimension $n$.

Proof. Let $x$ be a contact point, that is, $x \in\{u=\Gamma\} \subset B_{R}$. From Lemma2.6, $\mathcal{M}^{+} u(x)$ can be defined classically and we have

$$
\mathcal{M}^{+} u(x)=(2-\sigma) \int_{\mathbb{R}^{n}}\left\{\Lambda \mu^{+}(u, x, z)-\lambda \mu^{-}(u, x, z)\right\} \frac{l(|z|)}{|z|^{n}} d z \geq-f(x),
$$

where $\mu(u, x, z)=u(x+z)+u(x-z)-2 u(x)$, and $\mu^{ \pm}(u, x, z)=\max \{ \pm \mu(u, x, z), 0\}$.

We note that $u(x)=\Gamma(x)>0$. If $x+z \in B_{3 R}$ and $x-z \in B_{3 R}$, then we have $\mu(u, x, z) \leq 0$ since $\Gamma$ lies above $u$. If $x+z \notin B_{3 R}$, then $x+z$ and $x-z$ do not belong to $B_{R}$, which implies 
that $\mu(u, x, z) \leq 0$. Thus it follows that $\mu(u, x, z) \leq 0$ for any $z \in \mathbb{R}^{n}$ and hence

$$
\begin{aligned}
f(x) & \geq(2-\sigma) \lambda \int_{\mathbb{R}^{n}} \mu^{-}(u, x, z) \frac{l(|z|)}{|z|^{n}} d z \\
& \geq(2-\sigma) \lambda \int_{B_{r_{0}}(0)} \mu^{-}(u, x, z) \frac{l(|z|)}{|z|^{n}} d z=(2-\sigma) \lambda \sum_{k=0}^{+\infty} \int_{\mathcal{R}_{k}(0)} \mu^{-}(u, x, z) \frac{l(|z|)}{|z|^{n}} d z .
\end{aligned}
$$

The concavity of $\Gamma$ implies that if $u(x+z)<u(x)+z \cdot \nabla \Gamma(x)-M r_{k}^{2}$ for some $z \in B_{R}$, then $\mu^{-}(u, x, z) \geq M r_{k}^{2}$. Indeed, we have that $x \pm z \in B_{3 R}$ and

$$
\begin{aligned}
\mu(u, x, z) & =u(x+z)+u(x-z)-2 u(x) \leq u(x+z)+\Gamma(x-z)-2 u(x) \\
& <\left\{u(x)+z \cdot \nabla \Gamma(x)-M r_{k}^{2}\right\}+\{\Gamma(x)-z \cdot \nabla \Gamma(x)\}-2 u(x)=-M r_{k}^{2} .
\end{aligned}
$$

Suppose to the contrary that 12 is not true. Then we have

$$
\begin{aligned}
\frac{f(x)}{2-\sigma} & \geq \lambda \sum_{k=0}^{+\infty} \int_{\mathcal{R}_{k}(0)} \mu^{-}(u, x, z) \frac{l(|z|)}{|z|^{n}} d z=\lambda l(R) \sum_{k=0}^{+\infty} \int_{\mathcal{R}_{k}(0)} \mu^{-}(u, x, z) \frac{l(|z|)}{l(R)} \frac{1}{|z|^{n}} d z \\
& \geq \lambda l(R) \sum_{k=0}^{+\infty} \int_{\mathcal{R}_{k}(0)} \mu^{-}(u, x, z) \frac{1}{a_{0}}\left(\frac{|z|}{R}\right)^{-\sigma+\delta} \frac{1}{r_{k}^{n}} d z \\
& \geq \lambda l(R) \frac{1}{a_{0}} \sum_{k=0}^{+\infty}\left(\frac{r_{k}}{R}\right)^{-\sigma+\delta} \frac{1}{r_{k}^{n}} \int_{\mathcal{R}_{k}(0)} \mu^{-}(u, x, z) d z \\
& \geq \lambda l(R) \frac{1}{a_{0}} \sum_{k=0}^{+\infty}\left(\frac{r_{k}}{R}\right)^{-\sigma+\delta} \frac{1}{r_{k}^{n}} \frac{\tilde{C}}{l(R) R^{2}} \frac{f(x)}{M}\left|\mathcal{R}_{k}(x)\right| M r_{k}^{2} \\
& =\lambda l(R) \frac{1}{a_{0}} \sum_{k=0}^{+\infty}\left(\frac{r_{k}}{R}\right)^{-\sigma+\delta} \frac{r_{k}^{2}}{r_{k}^{n}} \frac{\tilde{C} f(x)}{l(R) R^{2}}\left|\mathcal{R}_{k}(0)\right|=\frac{\lambda c_{n}}{a_{0}} \tilde{C} f(x) \sum_{k=0}^{+\infty}\left(\frac{r_{k}}{R}\right)^{2-\sigma+\delta} \\
& \geq \frac{\lambda c_{n}}{a_{0}} \tilde{C} f(x) \sum_{k=0}^{+\infty}\left(\frac{r_{k}}{R}\right)^{2-\sigma+(2-\sigma) / 2} \geq \frac{\lambda c_{n}}{a_{0}} \tilde{C} f(x) \sum_{k=0}^{+\infty}\left(\frac{r_{k}}{R}\right)^{2(2-\sigma)} \\
& =\frac{\lambda c_{n}}{a_{0}} \tilde{C} f(x) \frac{\rho_{0}^{2(2-\sigma)}}{2\left(1-2^{-2(2-\sigma)}\right)} \geq \frac{\lambda c_{n}}{a_{0}} \tilde{C} f(x) \frac{\rho_{0}^{4}}{2\left(1-2^{-2(2-\sigma)}\right)}
\end{aligned}
$$

since $0 \leq \delta \leq(2-\sigma) / 2$. By choosing $\tilde{C} \geq a_{0} \frac{2\left(1-2^{-2(2-\sigma)}\right)}{\lambda c_{n} \rho_{0}^{4}(2-\sigma)}$ which is bounded above by a uniform constant for $\sigma \in\left[\sigma_{0}, 2\right)$, the result follows.

In the proof of Lemma 3.2 we observe that $f(x)$ is positive for $x \in\{u=\Gamma\}$.

Lemma 3.3. Under the same assumption as Lemma 3.2 there exists uniform constants $\epsilon_{n} \in(0,1)$ and $\tilde{M}:=\tilde{C} / \epsilon_{n}>0$ such that for each $x \in\{u=\Gamma\}$, we find some $r=r_{k} \leq$ $\rho_{0} 2^{-\frac{1}{2(2-\sigma)}} R$ which satisfies the following:

(a)

$$
\left|\left\{y \in B_{r}(x) \backslash B_{r / 2}(x): u(y)<u(x)+(y-x) \cdot \nabla \Gamma(x)-\frac{\tilde{M} f(x)}{l(R) R^{2}} r^{2}\right\}\right| \leq \epsilon_{n}\left|B_{r}(x) \backslash B_{r / 2}(x)\right|,
$$

(b)

$$
\Gamma(y) \geq u(x)+(y-x) \cdot \nabla \Gamma(x)-\frac{\tilde{M} f(x)}{l(R) R^{2}} r^{2} \quad \forall y \in B_{r / 2}(x),
$$


(c)

$$
\left|\nabla \Gamma\left(B_{r / 4}(x)\right)\right| \leq c_{n}\left(\frac{\tilde{M} f(x)}{l(R) R^{2}}\right)^{n}\left|B_{r / 4}(x)\right|
$$

where $\tilde{C}>0$ is the uniform constant as in Lemma 3.2 and $\epsilon_{n} \in(0,1)$ and $c_{n}>0$ are uniform constant depending only on $n$.

Proof. For a small $\epsilon_{n}>0$, let $\tilde{M}:=\tilde{C} / \epsilon_{n}$. We apply Lemma3.2 with $M=\tilde{M} \frac{f(x)}{l(R) R^{2}}$ to have

$$
\left|\left\{y \in B_{r}(x) \backslash B_{r / 2}(x): u(y)<u(x)+(y-x) \cdot \nabla \Gamma(x)-\frac{\tilde{M} f(x)}{l(R) R^{2}} r^{2}\right\}\right| \leq \epsilon_{n}\left|B_{r}(x) \backslash B_{r / 2}(x)\right|
$$

for some $r=r_{k}$, which proves (a). With the help of (a), we employ the same arguments as the proofs of Lemma 8.4 and Corollary 8.5 in [CS1] to show (b) and (c).

Now we obtain a nonlocal version of the ABP estimate in the following theorem making use of Lemma 3.3 together with a dyadic cube decomposition; we refer to [CS1, Theorem 8.7] for the proof.

Theorem 3.4 (ABP type estimate). Let $R \in(0,1)$, and let $\rho_{0} \in(0,1 /(32 \sqrt{n})]$ be a constant. Let $u$ be a viscosity subsolution of

$$
\mathcal{M}_{\mathfrak{Q}(\lambda, \Lambda, l)}^{+} u=-f \quad \text { on } B_{R}
$$

such that $u \leq 0$ in $\mathbb{R}^{n} \backslash B_{R}$, and let $\Gamma$ be the concave envelope of $u^{+}$in $B_{3 R}$. Then there exists a finite, disjoint family of open cubes $Q_{j}$ with diameters $d_{j} \leq \rho_{0} 2^{-\frac{1}{2(2-\sigma)} R}$ such that $\left\{\bar{Q}_{j}\right\}$ covers the contact set $\{u=\Gamma\}$, and satisfies the following:

(a) $\{u=\Gamma\} \cap \bar{Q}_{j} \neq \emptyset$ for any $Q_{j}$

(b) $\left|\nabla \Gamma\left(\bar{Q}_{j}\right)\right| \leq c_{n}\left(\frac{\tilde{C}}{l(R) R^{2}}\right)^{n}\left(\max _{\bar{Q}_{j} \cap\{u=\Gamma\}} f^{n}\right)\left|Q_{j}\right|$,

(c) $\left|\left\{y \in 32 \sqrt{n} Q_{j}: u(y) \geq \Gamma(y)-c_{n} \frac{\tilde{C}}{l(R) R^{2}}\left(\max _{\bar{Q}_{j} \cap\{u=\Gamma\}} f\right) d_{j}^{2}\right\}\right| \geq \mu\left|Q_{j}\right|$

for $\mu:=1-\epsilon_{n} \in(0,1)$, where $\tilde{C}>0$ is the uniform constant as in Lemma 3.2 and $\epsilon_{n} \in(0,1)$ (appearing in Lemma 3.3) and $c_{n}>0$ are uniform constant depending only on $n$.

3.2. Barrier function. As in [CS1] and [KL2], we construct the barrier function at each scale, where the monotone function $L$ associated with $l$ given in Property 1.1 plays a role to obtain scale invariant estimates.

Lemma 3.5. Let $R \in(0,1 / 2)$. For $\kappa_{1} \in(0,1)$, there exist uniform constants $p=p(n, \lambda, \Lambda)>$ $n$, and $\epsilon_{0} \in(0,1 / 8)$ such that the function $\varphi(x):=\min \left\{\left|\kappa_{0} R\right|^{-p},|x|^{-p}\right\}$ for $\kappa_{0}:=\epsilon_{0} \kappa_{1}>0$ satisfies

$$
\mathcal{M}_{\mathfrak{L}(\lambda, \Lambda, l)}^{-} \varphi(x) \geq 0, \quad \forall x \in B_{R} \backslash B_{\kappa_{1} R}
$$


Proof. Assume without loss of generality that $x=R_{0} e_{1}$ for $\kappa_{1} R \leq R_{0}<R$. We need to compute

$$
\begin{aligned}
\mathcal{M}^{-} \varphi(x) & =(2-\sigma) \int_{\mathbb{R}^{n}}\left\{\lambda \mu^{+}(\varphi, x, y)-\Lambda \mu^{-}(\varphi, x, y)\right\} \frac{l(|y|)}{|y|^{n}} d y \\
& =(2-\sigma) \int_{\mathbb{R}^{n}} \frac{\lambda \mu^{+}}{2} \frac{l(|y|)}{|y|^{n}} d y+(2-\sigma) \int_{B_{\rho_{1} R_{0}}}\left(\frac{\lambda}{2} \mu^{+}-\Lambda \mu^{-}\right) \frac{l(|y|)}{|y|^{n}} d y \\
& +(2-\sigma) \int_{B_{\rho_{1} R_{0}}^{c}}\left(\frac{\lambda}{2} \mu^{+}-\Lambda \mu^{-}\right) \frac{l(|y|)}{|y|^{n}} d y \\
& \geq(2-\sigma) \int_{\mathbb{R}^{n}} \frac{\lambda \mu^{+}}{2} \frac{l(|y|)}{|y|^{n}} d y+(2-\sigma) \int_{B_{\rho_{1} R_{0}}}\left(\frac{\lambda}{2} \mu^{+}-\Lambda \mu^{-}\right) \frac{l(|y|)}{|y|^{n}} d y \\
& -2(2-\sigma) \Lambda R_{0}^{-p} \int_{B_{\rho_{1} R_{0}}^{c}} \frac{l(|y|)}{|y|^{n}} d y=: I_{1}+I_{2}+I_{3},
\end{aligned}
$$

where $\rho_{1} \leq \min \{\rho, 1 / 2\}$ will be chosen sufficiently small later .

For $|y|<\frac{R_{0}}{2}$, we have

$$
\begin{aligned}
|x+y|^{-p}+|x-y|^{-p}-2|x|^{-p} & =R_{0}^{-p}\left\{\left|\frac{x}{R_{0}}+\frac{y}{R_{0}}\right|^{-p}+\left|\frac{x}{R_{0}}-\frac{y}{R_{0}}\right|^{-p}-2\right\} \\
& \geq R_{0}^{-p} p\left\{-|\bar{y}|^{2}+(p+2) \bar{y}_{1}^{2}-\frac{1}{2}(p+2)(p+4) \bar{y}_{1}^{2}|\bar{y}|^{2}\right\}
\end{aligned}
$$

for $\bar{y}:=y / R_{0}$; see [CS1, Lemma 9.1]. We choose $\mathbb{N} \ni p>n$ large enough so that

$$
(p+2) \frac{\lambda}{2} \int_{\partial B_{1}} y_{1}^{2} d \sigma(y)-\Lambda\left|\partial B_{1}\right|=: \bar{\delta}>0 .
$$

We use (13), (14), Lemma 3.1 and Property 1.1 to obtain

$$
\begin{aligned}
I_{2} & =(2-\sigma) \int_{B_{\rho_{1} R_{0}}}\left(\frac{\lambda}{2} \mu^{+}-\Lambda \mu^{-}\right) \frac{l(|y|)}{|y|^{n}} d y \\
& \geq(2-\sigma) p R_{0}^{-p} \int_{B_{\rho_{1} R_{0}}}\left\{\frac{\lambda}{2}(p+2) \frac{y_{1}^{2}}{R_{0}^{2}}-\Lambda\left(\frac{|y|^{2}}{R_{0}^{2}}+\frac{(p+2)(p+4)}{2} \frac{y_{1}^{2}|y|^{2}}{R_{0}^{4}}\right)\right\} \frac{l(|y|)}{|y|^{n}} d y \\
& \geq(2-\sigma) p R_{0}^{-p}\left\{\frac{\bar{\delta}}{R_{0}^{2}} \int_{0}^{\rho_{1} R_{0}} s l(s) d s-\frac{\Lambda(p+2)(p+4) \omega_{n}}{2 R_{0}^{4}} \int_{0}^{\rho_{1} R_{0}} s^{3} l(s) d s\right\} \\
& \geq(2-\sigma) p R_{0}^{-p}\left\{\frac{\bar{\delta}}{2 a_{0}(2-\sigma)} \rho_{1}^{2} l\left(\rho_{1} R_{0}\right)-\frac{\Lambda(p+2)(p+4) \omega_{n}}{2} a_{0} \rho_{1}^{4} l\left(\rho_{1} R_{0}\right)\right\} \\
& =p R_{0}^{-p}\left\{\frac{\bar{\delta}}{2 a_{0}} \rho_{1}^{2}-(2-\sigma) \frac{\Lambda(p+2)(p+4) \omega_{n}}{2} a_{0} \rho_{1}^{4}\right\} l\left(\rho_{1} R_{0}\right) \\
& \geq \frac{p R_{0}^{-p}}{2}\left\{\frac{\bar{\delta}}{2 a_{0}} \rho_{1}^{2}-(2-\sigma) \frac{\Lambda(p+2)(p+4) \omega_{n}}{2} a_{0}\right\} L\left(\rho_{1} R_{0}\right) .
\end{aligned}
$$

We select a uniform constant $\rho_{1}=\rho_{1}\left(a_{0}, a_{\infty}, \sigma_{0}\right) \leq \min (\rho, 1 / 2)$ small so that

$$
2 a_{\infty} \leq \frac{1}{2 a_{0}^{2}}\left(\rho_{1}^{-\sigma_{0} / 2}-1\right) \leq L\left(\rho_{1}\right)
$$


and then we use Lemma 3.1 and Properties 1.1 and 1.2 again to have

$$
\begin{aligned}
-I_{3} & =2(2-\sigma) \Lambda R_{0}^{-p} \int_{B_{\rho_{1} R_{0}}^{c}} \frac{l(|y|)}{|y|^{n}} d y \leq 2(2-\sigma) \Lambda R_{0}^{-p} \omega_{n}\left\{\frac{2 a_{\infty}}{\sigma}+\frac{1}{\sigma} L\left(\rho_{1} R_{0}\right)\right\} \\
& \leq \frac{2-\sigma}{\sigma} 2 \Lambda \omega_{n} R_{0}^{-p}\left\{\frac{1}{2 a_{0}^{2}}\left(\rho_{1}^{-\sigma_{0} / 2}-1\right)+L\left(\rho_{1} R_{0}\right)\right\} \leq \frac{2-\sigma}{\sigma_{0}} 4 \Lambda \omega_{n} R_{0}^{-p} L\left(\rho_{1} R_{0}\right),
\end{aligned}
$$

where we note that $L$ is monotone. Thus we deduce that

$$
\begin{aligned}
I_{2}+I_{3} & \geq \frac{p R_{0}^{-p}}{2}\left\{\frac{\bar{\delta}}{2 a_{0}} \rho_{1}^{2}-(2-\sigma) \frac{\Lambda(p+2)(p+4) \omega_{n} a_{0}}{2}\right\} L\left(\rho_{1} R_{0}\right)-\frac{2-\sigma}{\sigma_{0}} 4 \Lambda \omega_{n} R_{0}^{-p} L\left(\rho_{1} R_{0}\right) \\
& =\frac{R_{0}^{-p}}{2} L\left(\rho_{1} R_{0}\right)\left\{\frac{p \bar{\delta}}{2 a_{0}} \rho_{1}^{2}-(2-\sigma) \frac{\Lambda p(p+2)(p+4) \omega_{n} a_{0}}{2}-(2-\sigma) \frac{8 \Lambda \omega_{n}}{\sigma_{0}}\right\} \\
& \geq 0
\end{aligned}
$$

for any $\sigma \in\left[\sigma_{1}, 2\right)$, where $\sigma_{1} \in\left[\sigma_{0}, 2\right)$ depends only on $n, \lambda, \Lambda, a_{0}, a_{\infty}, \rho$, and $\sigma_{0}$. Thus the lemma holds true for $\sigma \in\left[\sigma_{1}, 2\right)$.

For $\sigma \in\left[\sigma_{0}, \sigma_{1}\right)$, we will make $I_{1}$ sufficiently large by selecting $\kappa_{0}>0$ small. For $x=R_{0} e_{1}$ with $\kappa_{1} R \leq R_{0}<R$, we have that for $\kappa_{0}:=\epsilon_{0} \kappa_{1} \in\left(0, \kappa_{1} / 8\right)$

$$
\begin{aligned}
I_{1} & \geq\left(2-\sigma_{1}\right) \int_{\mathbb{R}^{n}} \frac{\lambda \mu^{+}}{2} \frac{l(|y|)}{|y|^{n}} d y \\
& \geq\left(2-\sigma_{1}\right) \frac{\lambda}{2} \int_{B_{R_{0} / 4}(x)}\left\{|x-y|^{-p}-2 R_{0}^{-p} \frac{l(|y|)}{|y|^{n}} d y\right. \\
& \geq\left(2-\sigma_{1}\right) \frac{\lambda}{4} \int_{B_{R_{0} / 4}(x) \backslash B_{\kappa_{0} R}(x)}|x-y|^{-p} \frac{l(|y|)}{|y|^{n}} d y=\left(2-\sigma_{1}\right) \frac{\lambda}{4} \int_{B_{R_{0} / 4}(0) \backslash B_{\kappa_{0} R} R}|z|^{-p} \frac{l(|x+z|)}{|x+z|^{n}} d z \\
& \left.\geq\left(2-\sigma_{1}\right) \frac{\lambda}{2^{n+2} R_{0}^{n}} \int_{s \in\left[R_{0} / 2,3 R_{0} / 2\right]} l(s)\right) \omega_{n} \int_{\kappa_{0} R}^{R_{0} / 4} s^{-p+n-1} d s \\
& \geq\left(2-\sigma_{1}\right) \frac{\lambda}{2^{n+2} R_{0}^{n}} \frac{1}{p-n}\left\{\left(\kappa_{0} R\right)^{-p+n}-\left(R_{0} / 4\right)^{-p+n}\right\} \min _{s \in\left[R_{0} / 2,3 R_{0} / 2\right]} l(s) \\
& \geq\left(2-\sigma_{1}\right) \frac{\lambda}{2^{n+2} R_{0}^{n}} \frac{R_{0}^{-p+n}}{p-n}\left\{\left(\frac{\kappa_{1}}{\kappa_{0}}\right)^{p-n}-4^{p-n}\right\} \min _{s \in\left[R_{0} / 2,3 R_{0} / 2\right]} l(s) \\
& \geq\left(2-\sigma_{1}\right) \frac{\lambda}{2^{n+3} R_{0}^{n}} \frac{R_{0}^{-p+n}}{p-n}\left(\frac{\kappa_{1}}{\kappa_{0}}\right)^{p-n} \min _{s \in\left[R_{0} / 2,3 R_{0} / 2\right]} l(s) \\
& \geq\left(2-\sigma_{1}\right) \frac{\lambda}{2^{n+3} R_{0}^{n}} \frac{R_{0}^{-p+n}}{p-n} \epsilon_{0}^{-p+n} \frac{1}{a_{0}}\left(\frac{2 \rho_{1}}{3}\right)^{\sigma+\delta} l\left(\rho_{1} R_{0}\right) \\
& \geq\left(2-\sigma_{1}\right) \frac{\lambda}{2^{n+3}} \frac{R_{0}^{-p}}{p-n} \frac{1}{\epsilon_{0}} \frac{1}{a_{0}}\left(\frac{2 \rho_{1}}{3}\right)^{3} \frac{1}{2} L\left(\rho_{1} R_{0}\right) .
\end{aligned}
$$

From the argument above, we notice that for $\sigma \in\left[\sigma_{0}, 2\right)$,

$$
I_{2}+I_{3} \geq-C R_{0}^{-p} L\left(\rho_{1} R_{0}\right)
$$

where a uniform constant $C>0$ depends only on $n, \lambda, \Lambda, a_{0}, a_{\infty}, \rho$, and $\sigma_{0}$. Therefore, we choose a uniform constant $\epsilon_{0}=\frac{\kappa_{0}}{\kappa_{1}} \in(0,1 / 8)$ sufficiently small to conclude that

$$
\mathcal{M}^{-} \varphi(x) \geq I_{1}+I_{2}+I_{3} \geq 0
$$

for $x \in B_{R} \backslash B_{\kappa_{1} R}$ in the case when $\sigma \in\left[\sigma_{0}, \sigma_{1}\right)$. This finishes the proof. 
Lemma 3.6. Let $R \in(0,1 / 2)$ and $0<\delta_{1}<\delta_{2}<1$. Assume $\delta_{1} \leq \rho_{1}$, where $\rho_{1}=$ $\rho_{1}\left(a_{0}, a_{\infty}, \rho, \sigma_{0}\right) \leq \min (\rho, 1 / 2)$ is the constant satisfying (15). There exists a continuous function $\Phi$ in $\mathbb{R}^{n}$ such that

(a) $\Phi$ is nonnegative and uniformly bounded in $\mathbb{R}^{n}$,

(b) $\Phi=0$ outside $B_{R}$,

(c) $\Phi \geq 2$ in $B_{\delta_{2} R}$,

(d) $L\left(\delta_{1} R\right)^{-1} \mathcal{M}_{\mathfrak{Q}(\lambda, \Lambda, l)}^{-} \Phi \geq-\psi$ in $\mathbb{R}^{n}$ for some nonnegative, uniformly bounded function $\psi$ such that $\operatorname{supp}(\psi) \subset B_{\delta_{1} R}$.

Proof. Let $\kappa_{1}:=\delta_{1} / 2$. According to Lemma 3.5, the function $\varphi(x):=\min \left\{\left|\kappa_{0} R\right|^{-p},|x|^{-p}\right\}$ satisfies

$$
\mathcal{M}^{-} \varphi(x) \geq 0 \quad \forall x \in B_{R} \backslash B_{\kappa_{1} R}
$$

for some $0<\kappa_{0}<\kappa_{1} / 8$ and $p>n$. Now we define $\Phi: \mathbb{R}^{n} \rightarrow[0,+\infty)$ by

$$
\Phi(x):=c_{0}\left\{\begin{array}{lr}
P(x) & \forall x \in B_{\kappa_{0} R} \\
\left(\kappa_{0} R\right)^{p}\left\{\min \left(\left|\kappa_{0} R\right|^{-p},|x|^{-p}\right)-R^{-p}\right\} & \forall x \in B_{R} \backslash B_{\kappa_{0} R} \\
0 & \text { outside } B_{R},
\end{array}\right.
$$

where $P(x):=-a|x|^{2}+b$ with $a=\frac{1}{2} p\left(\kappa_{0} R\right)^{-2}$ and $b:=1-\kappa_{0}^{p}+\frac{1}{2} p$. Thus $\Phi$ is a $C^{1,1}$-function on $B_{R}$. By setting $c_{0}:=\frac{2}{\kappa_{0}^{p}\left(\delta_{2}^{-p}-1\right)}$, the property (b) follows. Note that Lemma 3.5 implies that

$$
\mathcal{M}^{-} \Phi(x) \geq 0 \quad \forall x \in B_{R} \backslash B_{\delta_{1} R / 2} .
$$

It remains to show that

$$
L\left(\delta_{1} R\right)^{-1} \mathcal{M}^{-} \Phi \geq-C \quad \text { in } B_{\delta_{1} R} .
$$

We use Properties 1.1 and 1.2 and Lemma 3.1 to deduce that for $x \in B_{\delta_{1} R}$,

$$
\begin{aligned}
\mathcal{M}^{-} \Phi(x) & \geq-(2-\sigma) \Lambda \int_{\mathbb{R}^{n}} \mu^{-}(\Phi, x, y) \frac{l(|y|)}{|y|^{n}} d y \\
& \geq-(2-\sigma) \Lambda \int_{B_{\delta_{1} R}} \mu^{-}(\Phi, x, y) \frac{l(|y|)}{|y|^{n}} d y-(2-\sigma) \Lambda 2 c_{0} b \int_{\mathbb{R}^{n} \backslash B_{\delta_{1} R}} \frac{l(|y|)}{|y|^{n}} d y \\
& \geq-(2-\sigma) \Lambda \int_{B_{\delta_{1} R}} \mu^{-}(\Phi, x, y) \frac{l(|y|)}{|y|^{n}} d y-\Lambda 4 c_{0} b \omega_{n} \frac{1}{\sigma}\left\{L\left(\delta_{1} R\right)+2 a_{\infty}\right\} \\
& \geq-(2-\sigma) \Lambda c_{1} R^{-2} \omega_{n} 2 a_{0} \frac{\delta_{1}^{2} R^{2}}{2-\sigma} l\left(\delta_{1} R\right)-\frac{8 b c_{0} \Lambda \omega_{n}}{\sigma_{0}} L\left(\delta_{1} R\right) \\
& \geq-\Lambda \omega_{n}\left\{4 a_{0} c_{1} \delta_{1}^{2}+\frac{8 b c_{0}}{\sigma_{0}}\right\} L\left(\delta_{1} R\right)
\end{aligned}
$$

since $D^{2} \Phi \geq-c_{1} R^{-2} \mathbf{I}$ a.e. in $B_{2 \delta_{1} R}$ for some $c_{1}=c_{1}\left(\delta_{1}, \delta_{2}\right)>0$, where we recall that $0<\delta_{1} \leq \rho_{1}$, and $\rho_{1}=\rho_{1}\left(a_{0}, a_{\infty}, \rho, \sigma_{0}\right) \leq \min (\rho, 1 / 2)$ satisfies (15).

3.3. Power decay estimate of super-level sets. We use the ABP type estimate in Theorem 3.4 and the barrier functions constructed in Lemma 3.6 to obtain the measure estimates of super-level sets of the viscosity supersolutions to fully nonlinear elliptic integro-differential operators with respect to $\mathfrak{L}(\lambda, \Lambda, l)$.

Lemma 3.7. Let $0<R<1 / 2$ and let $Q_{r}=Q_{r}(0)$ denote a dyadic cube of side $r$ centered at 0 for $r>0$. There exist uniform constants $\varepsilon_{0}, \rho_{0}, \mu_{0} \in(0,1)$ and $M_{0}>1$, depending only on $n, \lambda, \Lambda, a_{0}, a_{\infty}, \rho$, and $\sigma_{0}$, such that if

(a) $u \geq 0$ in $\mathbb{R}^{n}$, 
(b) $\inf _{Q_{3 R}} u \leq 1$,

(c) $\mathcal{M}_{\mathfrak{Q}(\lambda, \Lambda, l)}^{-} u \leq \varepsilon_{0} L\left(\rho_{0} R\right)$ on $Q_{2 R}$ in the viscosity sense,

then

$$
\left|\left\{u \leq M_{0}\right\} \cap Q_{\frac{R}{2 \sqrt{n}}}\right|>\mu_{0}\left|Q_{\frac{R}{2 \sqrt{n}}}\right| .
$$

Proof. Let $\rho_{0} \in(0,1)$ be a constant to be chosen later depending only on $n$ and $\rho_{1}>0$, where the constant $\rho_{1}$ satisfies 15 . Let $\Phi$ be the barrier function in Lemma 3.6 with $\delta_{1}:=\rho_{0}\left(\leq \min \left\{1 /(32 \sqrt{n}), \rho_{1}\right\}\right)$, and $\delta_{2}:=\frac{3}{4}$. Then

$$
v:=\Phi-u
$$

satisfies that $v \leq 0$ outside $B_{R}, \max _{B_{R}} v \geq 1$ and

$$
\mathcal{M}_{\mathfrak{Q}(\lambda, \Lambda, l)}^{+} v \geq \mathcal{M}_{\mathfrak{Q}(\lambda, \Lambda, l)}^{-} \Phi-\mathcal{M}_{\mathfrak{Q}(\lambda, \Lambda, l)}^{-} u \geq-\left(\psi+\varepsilon_{0}\right) L\left(\rho_{0} R\right) \quad \text { in } B_{R}
$$

in the viscosity sense. For the concave envelope $\Gamma$ of $v^{+}$in $B_{3 R}$, Theorem 3.4 with the help of Property 1.1 yields that

$$
\begin{aligned}
\frac{1}{R} \leq \frac{1}{R} \max _{B_{R}} v & \leq c_{n}\left|\nabla \Gamma\left(B_{R}\right)\right|^{1 / n} \leq c_{n}\left(\sum_{j}\left|\nabla \Gamma\left(\bar{Q}_{j}\right)\right|\right)^{1 / n} \\
& \leq c_{n} \frac{\tilde{C}}{l(R) R^{2}} L\left(\rho_{0} R\right)\left(\sum_{j} \max _{\bar{Q}_{j}}\left(\psi+\varepsilon_{0}\right)^{n}\left|Q_{j}\right|\right)^{1 / n} \\
& \leq c_{n} \tilde{C} \frac{L\left(\rho_{0} R\right)}{l\left(\rho_{0} R\right) R^{2}} a_{0} \rho_{0}^{-\sigma-\delta}\left(\sum_{j} \max _{\bar{Q}_{j}}\left(\psi^{n}+\varepsilon_{0}^{n}\right)\left|Q_{j}\right|\right)^{1 / n} \\
& \leq c_{n} \tilde{C} \frac{a_{0}}{\rho_{0}^{2}} \frac{1}{R^{2}}\left(\sum_{j} \max _{\bar{Q}_{j}}\left(\psi^{n}+\varepsilon_{0}^{n}\right)\left|Q_{j}\right|\right)^{1 / n}
\end{aligned}
$$

so we have

$$
\frac{1}{R} \leq \frac{C}{R^{2}}\left(\sum_{j} \max _{\bar{Q}_{j}}\left(\psi^{n}+\varepsilon_{0}^{n}\right)\left|Q_{j}\right|\right)^{1 / n}
$$

for a uniform constant $C>0$ depending only on $n, \lambda, a_{0}, \rho$, and $\sigma_{0}$, Recalling that the nonnegative function $\psi$ is uniformly bounded with $\operatorname{supp} \psi \subset B_{\rho_{0} R}$ in Lemma 3.6 and $\sum_{j}\left|Q_{j}\right| \leq c_{n}\left|B_{R}\right|$, it follows that

$$
\frac{1}{R} \leq \frac{C \varepsilon_{0}}{R}+\frac{C}{R^{2}}\left(\sum_{Q_{j} \cap B_{\rho_{0} R} \neq \emptyset}\left|Q_{j}\right|\right)^{1 / n} .
$$

By selecting $\varepsilon_{0}>0$ small, we have

$$
\frac{C}{R}\left(\sum_{\bar{Q}_{j} \cap B_{\rho_{0} R} \neq \emptyset}\left|Q_{j}\right|\right)^{1 / n} \geq \frac{1}{2} .
$$


Now we select $\rho_{0}>0$ sufficiently small such that $32 \sqrt{n} \rho_{0} \leq \frac{1}{8 \sqrt{n}}$ in order to show that $32 \sqrt{n} Q_{j} \subset B_{\frac{R}{4 \sqrt{n}}}$ for any $Q_{j}$ satisfying $\bar{Q}_{j} \cap B_{\rho_{0} R} \neq \emptyset$. Thus $\bigcup \bar{Q}_{j}$ is covered by $\left\{32 \sqrt{n} Q_{j}: \bar{Q}_{j} \cap B_{\rho_{0} R} \neq \emptyset\right\}$ contained in $B_{\frac{R}{4 \sqrt{n}}}$.

$$
\bar{Q}_{j} \cap B_{\rho_{0} R} \neq \emptyset
$$

On the other hand, according to Theorem 3.4 together with the previous argument, we have

$$
\left|\left\{y \in 32 \sqrt{n} Q_{j}: u(y) \leq M_{0}\right\}\right| \geq \mu\left|Q_{j}\right|
$$

for some $M_{0}>1$. Indeed, from the previous argument, we see that

$$
c_{n} \frac{\tilde{C}}{l(R) R^{2}} L\left(\rho_{0} R\right) \max _{\bar{Q}_{j}}\left(\psi+\varepsilon_{0}\right) d_{j}^{2} \leq M_{1}
$$

for a uniform constant $M_{1}>1$ with respect to $\sigma \in\left[\sigma_{0}, 2\right)$ since $d_{j} \leq \rho_{0} R$. Then it follows from Theorem 3.4 that

$$
\begin{aligned}
\mu\left|Q_{j}\right| & \leq\left|\left\{y \in 32 \sqrt{n} Q_{j}: v(y) \geq \Gamma(y)-M_{1}\right\}\right| \\
& \leq\left|\left\{y \in 32 \sqrt{n} Q_{j}: u(y) \leq\|\Phi\|_{L^{\infty}\left(\mathbb{R}^{n}\right)}+M_{1}=: M_{0}\right\}\right|
\end{aligned}
$$

since $\Phi$ is uniformly bounded in $\mathbb{R}^{n}$, and $\Gamma$ is positive in $B_{3 R}$. Taking a subcover of $\left\{32 \sqrt{n} Q_{j}: \bar{Q}_{j} \cap B_{\rho_{0} R} \neq \emptyset\right\}$ with finite overlapping, we deduce from (16) and (17) that for uniform constants $M_{0}>1$ and $\mu_{0} \in(0,1)$,

$$
\left|\left\{u \leq M_{0}\right\} \cap Q_{\frac{R}{2 \sqrt{n}}}\right| \geq\left|\left\{u \leq M_{0}\right\} \cap B_{\frac{R}{4 \sqrt{n}}}\right|>\mu_{0}\left|Q_{\frac{R}{2 \sqrt{n}}}\right|,
$$

which finishes the proof.

The Calderón-Zygmund technique combined with Lemma 3.7 implies the following decay measure estimate of super-level sets making use of the monotonicity of the function $L$.

Corollary 3.8. Under the same assumption as Lemma 3.7 we have

$$
\left|\left\{u>M_{0}^{k}\right\} \cap Q_{\frac{R}{2 \sqrt{n}}}\right| \leq\left(1-\mu_{0}\right)^{k}\left|Q_{\frac{R}{2 \sqrt{n}}}\right|, \quad \forall k=1,2, \cdots,
$$

and hence

$$
\left|\{u>t\} \cap Q_{\frac{R}{2 \sqrt{n}}}\right| \leq C R^{n} t^{-\epsilon} \quad \forall t>0,
$$

where $C>0$ and $\epsilon>0$ are uniform constants depending only on $n, \lambda, \Lambda, a_{0}, a_{\infty}, \rho$, and $\sigma_{0}$.

Using a standard covering argument, we deduce the weak Harnack inequality as follows.

Theorem 3.9 (Weak Harnack inequality). For $0<R<1$, and $C_{0}>0$, let $u$ be a nonnegative function in $\mathbb{R}^{n}$ such that

$$
\mathcal{M}_{\mathfrak{Q}(\lambda, \Lambda, l)}^{-} u \leq C_{0} \quad \text { in } B_{2 R}
$$

in the viscosity sense. Then we have

$$
\left|\{u>t\} \cap B_{R}\right| \leq C R^{n}\left(u(0)+\frac{C_{0}}{L\left(\rho_{0} R\right)}\right)^{\epsilon} t^{-\epsilon} \quad \forall t>0,
$$

and hence

$$
\left(f_{B_{R}}|u|^{p}\right)^{1 / p} \leq C\left\{u(0)+\frac{C_{0}}{L\left(\rho_{0} R\right)}\right\}
$$


where $C>0, \epsilon>0, \rho_{0} \in(0,1)$, and $p>0$ are uniform constants depending only on $n, \lambda, \Lambda$, $a_{0}, a_{\infty}, \rho$ and $\sigma_{0}$.

3.4. Harnack inequality. Making use of the weak Harnack inequality in Theorem 3.9. we prove the scale invariant Harnack inequality for fully nonlinear elliptic integro-differential operators with respect to $\mathfrak{L}(\lambda, \Lambda, l)$, where the constant in the Harnack estimate depends only on on $n, \lambda, \Lambda, a_{0}, a_{\infty}, \rho$ (in Properties 1.1 and 1.2) and $\sigma_{0}$. The proof of [CS1] Theorem 11.1] has been adapted to our elliptic integro-differential operators associated with regularly varying kernels at zero and infinity.

Theorem 3.10. For $0<R<1$, and $C_{0}>0$, let $u \in C\left(B_{2 R}\right)$ be a nonnegative function in $\mathbb{R}^{n}$ such that

$$
\mathcal{M}_{\mathfrak{Q}(\lambda, \Lambda, l)}^{-} u \leq C_{0} L\left(\rho_{0} R\right), \quad \text { and } \quad \mathcal{M}_{\mathfrak{Q}(\lambda, \Lambda, l)}^{+} u \geq-C_{0} L\left(\rho_{0} R\right) \quad \text { in } B_{2 R}
$$

in the viscosity sense, where $\rho_{0} \in(0,1)$ is the constant as in Theorem 3.9. Then we have

$$
\sup _{B_{\frac{R}{2}}} u \leq C\left(u(0)+C_{0}\right)
$$

where a uniform constant $C>0$ depends only on $n, \lambda, \Lambda, a_{0}, a_{\infty}, \rho$ and $\sigma_{0}$.

Proof. We may assume that $u>0, u(0) \leq 1$, and $C_{0}=1$. Let $\epsilon>0$ be the constant as in Theorem 3.9 and let $\gamma:=(n+2) / \epsilon$. Consider the minimal value of $\alpha>0$ such that

$$
u(x) \leq h_{\alpha}(x):=\alpha\left(1-\frac{|x|}{R}\right)^{-\gamma} \quad \forall x \in B_{R} .
$$

We claim that $\alpha>0$ is uniformly bounded. Let $x_{0}$ be a point such that $u\left(x_{0}\right)=h_{\alpha}\left(x_{0}\right)$. We may assume that $x_{0} \in B_{R}$, otherwise $\alpha$ is small. Let $d:=R-\left|x_{0}\right|$ and $r:=d / 2$.

Let $A:=\left\{u>u\left(x_{0}\right) / 2\right\}$. According to the weak Harnack inequality in Theorem 3.9 we have

$$
\left|A \cap B_{R}\right| \leq C R^{n}\left(\frac{2}{u\left(x_{0}\right)}\right)^{\epsilon} \leq C R^{n} \alpha^{-\epsilon}\left(\frac{d}{R}\right)^{\gamma \epsilon}=C \alpha^{-\epsilon}\left(\frac{d}{R}\right)^{3} d^{n} \leq C \alpha^{-\epsilon} d^{n} .
$$

This implies that

$$
\left|\left\{u>u\left(x_{0}\right) / 2\right\} \cap B_{r}\left(x_{0}\right)\right| \leq C \alpha^{-\epsilon}\left|B_{r}\left(x_{0}\right)\right|
$$

since $B_{r}\left(x_{0}\right) \Subset B_{R}$ and $r=d / 2$.

Now we will show that there is a uniform number $\theta \in(0,1)$ such that

$$
\left|\left\{u<u\left(x_{0}\right) / 2\right\} \cap B_{\theta r}\left(x_{0}\right)\right| \leq \frac{1}{2}\left|B_{\theta r}\left(x_{0}\right)\right|
$$

for a large constant $\alpha>1$, from which (18) yields that $\alpha>0$ is uniformly bounded. We first notice that for $x \in B_{\theta r}\left(x_{0}\right)$,

$$
u(x) \leq h_{\alpha}(x) \leq \alpha\left(\frac{d-\theta r}{R}\right)^{-\gamma}=\alpha\left(\frac{d}{R}\right)^{-\gamma}\left(1-\frac{\theta}{2}\right)^{-\gamma}=\left(1-\frac{\theta}{2}\right)^{-\gamma} u\left(x_{0}\right) .
$$

For $\theta \in(0,1)$, consider

$$
v(x):=\left(1-\frac{\theta}{2}\right)^{-\gamma} u\left(x_{0}\right)-u(x) .
$$

Note that $v$ is nonnegative in $B_{\theta r}\left(x_{0}\right)$. To apply the weak Harnack inequality to

$$
w:=v^{+},
$$


we will compute $\mathcal{M}^{-} w$ in $B_{\theta r}\left(x_{0}\right)$. First, we see that for $x \in B_{\theta r}\left(x_{0}\right)$,

$$
\begin{aligned}
\mathcal{M}^{-} w(x) & =(2-\sigma) \int_{\mathbb{R}^{n}}\left\{\lambda \mu^{+}\left(v^{+}, x, y\right)-\Lambda \mu^{-}\left(v^{+}, x, y\right)\right\} \frac{l(|y|)}{|y|^{n}} d y \\
& \leq \mathcal{M}^{-} v(x)+(2-\sigma) \int_{\mathbb{R}^{n}}\left\{\Lambda v^{-}(x+y)+\Lambda v^{-}(x-y)\right\} \frac{l(|y|)}{|y|^{n}} d y \\
& \leq L\left(\rho_{0} R\right)+(2-\sigma) \int_{\mathbb{R}^{n}}\left\{\Lambda v^{-}(x+y)+\Lambda v^{-}(x-y)\right\} \frac{l|y|)}{|y|^{n}} d y \\
& =L\left(\rho_{0} R\right)+2(2-\sigma) \int_{\{v(x+y)<0\}}-\Lambda v^{-}(x+y) \frac{l(|y|)}{|y|^{n}} d y \\
& \leq L\left(\rho_{0} R\right)+2(2-\sigma) \Lambda \int_{\mathbb{R}^{n} \backslash B_{\theta r}\left(x_{0}-x\right)}\left\{u(x+y)-\left(1-\frac{\theta}{2}\right)^{-\gamma} u\left(x_{0}\right)\right\}^{+} \frac{l(|y|)}{|y|^{n}} d y
\end{aligned}
$$

in the viscosity sense, where $v$ satisfies $\mathcal{M}^{-} v=\mathcal{M}^{-}[-u] \leq L\left(\rho_{0} R\right)$ on $B_{2 R}$ in the viscosity sense.

Consider the largest number $\beta>0$ such that

$$
u(x) \geq g_{\beta}(x):=\beta\left(1-\frac{|4 x|^{2}}{R^{2}}\right)^{+},
$$

and let $x_{1} \in B_{\underline{R}}$ be a point such that $u\left(x_{1}\right)=g_{\beta}\left(x_{1}\right)$. We observe that $\beta \leq 1$ since $u(0) \leq 1$. Using Lemma3.1, we have

$$
\begin{aligned}
(2-\sigma) \int_{\mathbb{R}^{n}} \mu^{-}\left(u, x_{1}, y\right) \frac{l(|y|)}{|y|^{n}} d y & \leq(2-\sigma) \int_{\mathbb{R}^{n}} \mu^{-}\left(g_{\beta}, x_{1}, y\right) \frac{l(|y|)}{|y|^{n}} d y \\
& =(2-\sigma)\left\{\int_{B_{\rho_{0} R}} \mu^{-}\left(g_{\beta}, x_{1}, y\right) \frac{l(|y|)}{|y|^{n}} d y+\int_{\mathbb{R}^{n} \backslash B_{\rho_{0} R}} \mu^{-}\left(g_{\beta}, x_{1}, y\right) \frac{l(|y|)}{|y|^{n}} d y\right\} \\
& \leq C(2-\sigma) \beta \int_{B_{\rho_{0} R}} \frac{|y|^{2}}{R^{2}} \frac{l(|y|)}{|y|^{n}} d y+2(2-\sigma) \beta \int_{\mathbb{R}^{n} \backslash B_{\rho_{0} R}} \frac{l(|y|)}{|y|^{n}} d y \\
& \leq \frac{C \beta}{R^{2}} 2 a_{0} \rho_{0}^{2} R^{2} l\left(\rho_{0} R\right)+2 \beta \frac{2-\sigma}{\sigma}\left\{L\left(\rho_{0} R\right)+2 a_{\infty}\right\} \\
& \leq C \beta a_{0} \rho_{0}^{2} L\left(\rho_{0} R\right)+\frac{8 \beta}{\sigma_{0}} L\left(\rho_{0} R\right) \leq C \beta L\left(\rho_{0} R\right) \leq C L\left(\rho_{0} R\right),
\end{aligned}
$$

where we recall that $0<\rho_{0} \leq \rho_{1}$; see (15). Since $\mathcal{M}^{-} u \leq L\left(\rho_{0} R\right)$ on $B_{2 R}$ in the viscosity sense, it follows that

$$
(2-\sigma) \int_{\mathbb{R}^{n}} \mu^{+}\left(u, x_{1}, y\right) \frac{l(|y|)}{|y|^{n}} d y \leq C L\left(\rho_{0} R\right)
$$

which asserts that

$$
(2-\sigma) \int_{\mathbb{R}^{n}}\left\{u\left(x_{1}+y\right)-2\right\}^{+} \frac{l(|y|)}{|y|^{n}} d y \leq C L\left(\rho_{0} R\right),
$$

where we note that $u\left(x_{1}\right) \leq \beta \leq 1$ and $u\left(x_{1}-y\right)>0$ for any $y \in \mathbb{R}^{n}$. 
We may assume that $u\left(x_{0}\right) \geq 2$, otherwise $\alpha$ is uniformly bounded. In order to estimate $\mathcal{M}^{-} w$ in $B_{\frac{\theta}{2} r}\left(x_{0}\right)$, we consider that for $x \in B_{\frac{\theta}{2} r}\left(x_{0}\right)$

$$
\begin{aligned}
& (2-\sigma) \int_{\mathbb{R}^{n} \backslash B_{\theta r}\left(x_{0}-x\right)}\left\{u(x+y)-\left(1-\frac{\theta}{2}\right)^{-\gamma} u\left(x_{0}\right)\right\}^{+} \frac{l(|y|)}{|y|^{n}} d y \\
=(2-\sigma) & \int_{\mathbb{R}^{n} \backslash B_{\theta r}\left(x_{0}-x\right)}\left\{u\left(x_{1}+x+y-x_{1}\right)-\left(1-\frac{\theta}{2}\right)^{-\gamma} u\left(x_{0}\right)\right\}^{+} \frac{l\left(\left|x+y-x_{1}\right|\right)}{\left|x+y-x_{1}\right|^{n}} \\
& \cdot\left(\frac{\left|x+y-x_{1}\right|^{n}}{|y|^{n}} \frac{l(|y|)}{l\left(\left|x+y-x_{1}\right|\right)}\right) d y .
\end{aligned}
$$

Since we see that for $x \in B_{\frac{\theta}{2} r}\left(x_{0}\right)$ and $y \in \mathbb{R}^{n} \backslash B_{\theta r}\left(x_{0}-x\right)$,

$$
\frac{\left|x+y-x_{1}\right|^{n}}{|y|^{n}} \frac{l(|y|)}{l\left(\left|x+y-x_{1}\right|\right)} d y \leq a_{0} a_{\infty}\left(\frac{6 R}{\theta r}\right)^{n+\sigma+\max \left(\delta, \delta^{\prime}\right)},
$$

it follows from (19) that for $x \in B_{\frac{\theta}{2} r}\left(x_{0}\right)$

$$
\begin{aligned}
\mathcal{M}^{-} w(x) & \leq L\left(\rho_{0} R\right)+2 \Lambda a_{0} a_{\infty} 3^{n+3}\left(\frac{2 R}{\theta r}\right)^{n+\sigma+\max \left(\delta, \delta^{\prime}\right)} C L\left(\rho_{0} R\right) \\
& \leq C\left(\frac{2 R}{\theta r}\right)^{n+\sigma+\delta} L\left(\rho_{0} R\right) \leq C\left(\frac{2 R}{\theta r}\right)^{n+2} L\left(\rho_{0} \frac{\theta r}{2}\right)
\end{aligned}
$$

owing to monotonicity of the function $L$.

Now we apply the weak Harnack inequality to $w$ in $B_{\frac{\theta}{2} r}\left(x_{0}\right)$ to obtain that

$$
\begin{aligned}
& \left|\left\{u<\frac{u\left(x_{0}\right)}{2}\right\} \cap B_{\theta r / 4}\left(x_{0}\right)\right|=\left|\left\{w>\left(\left(1-\frac{\theta}{2}\right)^{-\gamma}-\frac{1}{2}\right) u\left(x_{0}\right)\right\} \cap B_{\theta r / 4}\left(x_{0}\right)\right| \\
& \leq C(\theta r)^{n}\left(w\left(x_{0}\right)+C\left(\frac{2 R}{\theta r}\right)^{n+\sigma+\delta}\right)^{\epsilon}\left(\left(1-\frac{\theta}{2}\right)^{-\gamma}-\frac{1}{2}\right)^{-\epsilon} u\left(x_{0}\right)^{-\epsilon} \\
& =C(\theta r)^{n}\left(\left(\left(1-\frac{\theta}{2}\right)^{-\gamma}-1\right) u\left(x_{0}\right)+C\left(\frac{2 R}{\theta r}\right)^{n+2}\right)^{\epsilon}\left(\left(1-\frac{\theta}{2}\right)^{-\gamma}-\frac{1}{2}\right)^{-\epsilon} u\left(x_{0}\right)^{-\epsilon} \\
& \leq C(\theta r)^{n}\left(\left(\left(1-\frac{\theta}{2}\right)^{-\gamma}-1\right)^{\epsilon}+C\left(\frac{2 R}{\theta r}\right)^{(n+2) \epsilon} \frac{1}{u\left(x_{0}\right)^{\epsilon}}\right) \\
& \leq C(\theta r)^{n}\left(\left(\left(1-\frac{\theta}{2}\right)^{-\gamma}-1\right)^{\epsilon}+C\left(\frac{2 R}{\theta r}\right)^{(n+2-\gamma) \epsilon}\left(\frac{\theta}{4}\right)^{-\gamma \epsilon} \alpha^{-\epsilon}\right) \\
& \leq C(\theta r)^{n}\left(\left(\left(1-\frac{\theta}{2}\right)^{-\gamma}-1\right)^{\epsilon}+\theta^{-\gamma \epsilon} \alpha^{-\epsilon}\right)
\end{aligned}
$$

since $u\left(x_{0}\right)=\alpha(R / 2 r)^{\gamma}$ and $\gamma=(n+2) / \epsilon$. We choose a uniform constant $\theta>0$ sufficiently small so that

$$
C(\theta r)^{n}\left(\left(1-\frac{\theta}{2}\right)^{-\gamma}-1\right)^{\epsilon} \leq \frac{1}{4}\left|B_{\theta r / 4}\left(x_{0}\right)\right| .
$$

If $\alpha>0$ is sufficiently large, then we have

$$
C(\theta r)^{n} \theta^{-\gamma \epsilon} \alpha^{-\epsilon} \leq \frac{1}{4}\left|B_{\theta r / 4}\left(x_{0}\right)\right|,
$$

which implies that

$$
\left|\left\{u<\frac{u\left(x_{0}\right)}{2}\right\} \cap B_{\theta r / 4}\left(x_{0}\right)\right| \leq \frac{1}{2}\left|B_{\theta r / 4}\left(x_{0}\right)\right| .
$$


On the other hand, according to [18), we have that for large $\alpha>0$

$$
\left|\left\{u>\frac{u\left(x_{0}\right)}{2}\right\} \cap B_{\theta r / 4}\left(x_{0}\right)\right| \leq C \alpha^{-\epsilon}\left|B_{\theta r / 4}\left(x_{0}\right)\right|<\frac{1}{2}\left|B_{\theta r / 4}\left(x_{0}\right)\right|,
$$

which is a contradiction. Therefore, we conclude that $\alpha>0$ is uniformly bounded and that $\sup u \leq \alpha 2^{\gamma}$, which completes the proof. $B_{\frac{R}{2}}$

3.5. Hölder continuity. From the Harnack inequality, we obtain the following Hölder regularity of the viscosity solutions to fully nonlinear elliptic integro-differential equations with respect to $\mathfrak{L}(\lambda, \Lambda, l)$.

Theorem 3.11 (Hölder continuity). For $0<R<1$, and $C_{0}>0$, let $u \in C\left(B_{2 R}\right)$ be a nonnegative function in $\mathbb{R}^{n}$ such that

$$
\mathcal{M}_{\mathfrak{Q}(\lambda, \Lambda, l)}^{-} u \leq C_{0} L\left(\rho_{0} R\right), \quad \text { and } \quad \mathcal{M}_{\mathfrak{Q}(\lambda, \Lambda, l)}^{+} u \geq-C_{0} L\left(\rho_{0} R\right) \quad \text { in } B_{2 R}
$$

in the viscosity sense. Then we have

$$
R^{\alpha}[u]_{\alpha, B_{R}} \leq C\left(\|u\|_{L^{\infty}\left(\mathbb{R}^{n}\right)}+C_{0}\right)
$$

where $[u]_{\alpha, B_{R}}$ stands for the $\alpha$-Hölder seminorm on $B_{R}$, and uniform constants $\rho_{0}, \alpha \in(0,1)$ and $C>0$ depend only on $n, \lambda, \Lambda, a_{0}, a_{\infty}, \rho$ and $\sigma_{0}$.

3.6. $C^{1, \alpha}$ estimate. In this subsection, we present an interior $C^{1, \alpha}$ estimate for viscosity solutions to the elliptic integro-differetial operators as a important consequence of the Hölder estimate. To apply the incremental quotients technique iteratively in the nonlocal setting, the cancellation condition (20) below for the kernels at infinity is assumed; refer to [CS1, Section 13]. For contants $\theta_{0}>0$ and $D_{0}>0$, we define $\mathfrak{I}_{1}\left(\lambda, \Lambda, l ; \theta_{0}, D_{0}\right)$ by the class of the following linear integro-differential operators with the kernels $K$ :

$$
\mathcal{L} u(x)=\int_{\mathbb{R}^{n}} \mu(u, x, y) K(y) d y,
$$

such that

and

$$
(2-\sigma) \lambda \frac{l(|y|)}{|y|^{n}} \leq K(y) \leq(2-\sigma) \Lambda \frac{l(|y|)}{|y|^{n}},
$$

$$
\int_{\mathbb{R}^{n} \backslash B_{\theta_{0}}} \frac{|K(y)-K(y-h)|}{|y|} d y \leq D_{0}, \quad \forall|h|<\frac{\theta_{0}}{2} .
$$

Theorem 3.12. There is a uniform constant $\theta_{0}>0$ (depending only on $n, \lambda, \Lambda, a_{0}, a_{\infty}, \rho$, $\left.\sigma_{0}\right)$ such that if $u \in C\left(B_{1}\right)$ is a bounded, nonnegative function in $\mathbb{R}^{n}$ such that $\mathcal{I} u=0$ in $B_{1}$ in the viscosity sense for an elliptic operator $\mathcal{I}$ with respect to $\mathfrak{I}_{1}\left(\lambda, \Lambda, l ; \theta_{0}, D_{0}\right)$, then we have

$$
\|u\|_{C^{1, \alpha}\left(B_{1 / 2}\right)} \leq C\left(\|u\|_{L^{\infty}\left(\mathbb{R}^{n}\right)}+|\mathcal{I} 0|\right)
$$

where $\alpha \in(0,1)$ and $C>0$ depend only on $n, \lambda, \Lambda, a_{0}, a_{\infty}, \rho, \sigma_{0}$, and $D_{0}$.

Making use of the incremental quotients, we obtain $C^{1, \alpha}$-estimate for nonlocal operators. The uniform Hölder estimate in Theorem 3.11 is applicable to

$$
w_{h}(x):=\frac{u(x+h)-u(x)}{|h|^{\alpha}}
$$

for any small vector $h \in \mathbb{R}^{n}$ when (20) holds. Indeed, we introduce the cut-off function $\eta$ supported in a smaller ball, and divide the incremental quotient $w_{h}$ into two functions $w_{h, 1}:=\eta w_{h}$ and $w_{h, 2}:=(1-\eta) w_{h}$. With the help of (20), we deal with the incremental 
quotient of the kernel $K$ replacing the incremental quotient of $u$ in order to show that $\left|\mathcal{L} w_{h, 2}\right|$ is bounded by $C\|u\|_{L^{\infty}\left(\mathbb{R}^{n}\right)}$. So we apply Theorem 3.11 to $w_{h, 1}$ to deduce $C^{2 \alpha}$ for the Hölder exponent $\alpha>0$ in Theorem 3.11 Employing the procedure [1/ $\alpha]$ times, it follows that $u$ is Lipschitz continuous. By applying the previous argument to the Lipschitz quotient of $u$, we deduce the uniform $C^{1, \alpha}$-estimate. Note that the $C^{1, \alpha}$-estimate is not scale-invariant since it relies on the values $\theta_{0}$ and $D_{0}$.

3.7. Truncated kernels at infinity. In this subsection, we are concerned with the elliptic integro-differential operators associated with the symmetric kernels satisfying Property 1.1 near zero which may not satisfy Property 1.2 at infinity. This subsection corresponds to [CS1, Section 14] which involves in the results of the fractional Laplacian type integrodifferential operators. Consider the linear integro-differential operator $\mathcal{L}$

$$
\mathcal{L} u(x)=\int_{\mathbb{R}^{n}} \mu(u, x, y) K(y) d y,
$$

with the nonnegative kernel $K$ which is split by

$$
K(y)=K_{1}(y)+K_{2}(y) \geq 0 \quad \text { in } \mathbb{R}^{n},
$$

where the linear integro-differential operator $\mathcal{L}_{1}$ with the kernel $K_{1}$ belongs to $\mathfrak{L}(\lambda, \Lambda, l)$, and $\left\|K_{2}\right\|_{L^{1}\left(\mathbb{R}^{n}\right)} \leq \kappa$ for $\kappa \geq 0$. For $\kappa \geq 0$, we denote by $\tilde{\mathfrak{I}}(\lambda, \Lambda, l, \kappa)$ the class of all the linear integro-differential operators above. Using Lemma 3.1, we see that the truncated kernel $K$ at infinity satisfying

$$
(2-\sigma) \lambda \frac{l(|y|)}{|y|^{n}} \chi_{B_{1}(0)} \leq K(y) \leq(2-\sigma) \Lambda \frac{l(|y|)}{|y|^{n}} \chi_{B_{1}(0)}
$$

is one of the typical kernels for the linear integro-differential operators belonging to the class $\tilde{\mathfrak{L}}\left(\lambda, \Lambda, l, 2(2-\sigma) a_{\infty} / \sigma\right)$. It is obvious that the larger class $\tilde{\mathfrak{L}}(\lambda, \Lambda, l, \kappa)$ coincides with $\mathfrak{L}(\lambda, \Lambda, l)$ for $\kappa=0$. The Pucci type extremal operators with respect to the class $\tilde{\mathfrak{L}}(\lambda, \Lambda, l, \kappa)$ are defined as

$$
\begin{aligned}
& \mathcal{M}_{\tilde{\mathfrak{L}}(\lambda, \Lambda, l, \kappa)}^{+} u:=\sup _{\mathcal{L} \in \tilde{\mathfrak{I}}(\lambda, \Lambda, l, \kappa)} \mathcal{L} u, \\
& \mathcal{M}_{\tilde{\mathfrak{L}}(\lambda, \Lambda, l, \kappa)}^{-} u:=\inf _{\mathcal{L} \in \tilde{\mathfrak{I}}(\lambda, \Lambda, l, \kappa)} \mathcal{L} u .
\end{aligned}
$$

The same argument as in [CS1, Lemma 14.1] provides the following lemma regarding the relation between the Pucci type operators with respect to the classes $\tilde{\mathfrak{L}}(\lambda, \Lambda, l, \kappa)$ and $\mathfrak{L}(\lambda, \Lambda, l)$.

Lemma 3.13. Let $u$ be a bounded function in $\mathbb{R}^{n}$ and $C^{1,1}$ at $x$. Then we have

$$
\mathcal{M}_{\mathfrak{I}(\lambda, \Lambda, l, \kappa)}^{-} u(x) \geq \mathcal{M}_{\mathfrak{L}(\lambda, \Lambda, l)}^{-} u(x)-4 \kappa\|u\|_{L^{\infty}\left(\mathbb{R}^{n}\right)},
$$

and

$$
\mathcal{M}_{\mathfrak{\mathbb { Q }}(\lambda, \Lambda, l, \kappa)}^{+} u(x) \leq \mathcal{M}_{\mathfrak{Q}(\lambda, \Lambda, l)}^{+} u(x)+4 \kappa\|u\|_{L^{\infty}\left(\mathbb{R}^{n}\right)} .
$$

Applying Theorem 3.11 combined with Lemma 3.13 we deduce the Hölder estimate for the elliptic integro-differential operators associated with truncated kernels at infinity.

Theorem 3.14. For $0<R<1$, and $C_{0}>0$, let $u \in C\left(B_{2 R}\right)$ be a bounded, nonnegative function in $\mathbb{R}^{n}$ such that

$$
\mathcal{M}_{\tilde{\mathfrak{L}}(\lambda, \Lambda, l, \kappa)}^{-} u \leq C_{0} L\left(\rho_{0} R\right), \quad \text { and } \quad \mathcal{M}_{\tilde{\mathfrak{L}}(\lambda, \Lambda, l, \kappa)}^{+} u \geq-C_{0} L\left(\rho_{0} R\right) \quad \text { in } B_{2 R}
$$

in the viscosity sense. Then we have

$$
R^{\alpha}[u]_{\alpha, B_{R}} \leq C\left\{(1+4 \kappa)\|u\|_{L^{\infty}\left(\mathbb{R}^{n}\right)}+C_{0}\right\},
$$


where uniform constants $\rho_{0}, \alpha \in(0,1)$ and $C>0$ depend only on $n, \lambda, \Lambda, a_{0}, a_{\infty}, \rho$ and $\sigma_{0}$.

4. UNIFORM REGULARITY ESTIMATES FOR CERTAIN INTEGRO-DIFFERENTIAL OPERATORS AS $\sigma \rightarrow 2-$

In this section, we study the uniform Harnack inequality and Hölder estimate for the elliptic integro-differential operators associated with the certain regularly varying kernels at zero, where the regularity estimates remain uniform as the order $\sigma \in(0,2)$ of the operator tends to 2. Consider a measurable function $l_{0}:(0,+\infty) \rightarrow(0,+\infty)$ which stays locally bounded away from 0 and $+\infty$, and is slowly varying at zero and infinity. We may assume that $l_{0}(1)=1$. For $\sigma \in(0,2)$, we define

$$
l_{\sigma}(r):=r^{-\sigma} l_{0}(r)^{2-\sigma}, \quad \forall r>0 .
$$

It is easy to check that the function $l_{\sigma}$ varies regularly at zero and infinity with index $-\sigma \in(-2,0)$. As seen in Subsection 1.2, let $\mathfrak{L}\left(\lambda, \Lambda, l_{\sigma}\right)$ denote the class of all linear integro-differential operators

$$
\mathcal{L} u(x)=\int_{\mathbb{R}^{n}} \mu(u, x, y) K(y) d y
$$

with the kernels $K$ satisfying

$$
(2-\sigma) \lambda \frac{l_{\sigma}(|y|)}{|y|^{n}} \leq K(y) \leq(2-\sigma) \Lambda \frac{l_{\sigma}(|y|)}{|y|^{n}},
$$

where $\mu(u, x, y):=u(x+y)+u(x-y)-2 u(x)$. For a given constant $\sigma_{0} \in(0,2)$ and a function $l_{0}$, the uniform Harnack inequality and Hölder estimate for fully nonlinear elliptic integro-differential operators with respect to the class $\mathfrak{L}\left(\lambda, \Lambda, l_{\sigma}\right)$ for $\sigma \in\left[\sigma_{0}, 2\right)$ are established. In fact, once it is proved that the function $l_{\sigma}$ satisfies Properties 1.1 and 1.2 with uniform constants $a_{0}, a_{\infty} \geq 1$ and $\rho \in(0,1)$ with respect to $\sigma \in\left[\sigma_{0}, 2\right)$, the uniform regularity estimates follow from the results of Section 3 Here, the regularity estimates depend only on $n, \lambda, \Lambda, \sigma_{0}$, and the given function $l_{0}$. Therefore, it suffices to prove the following proposition in order to obtain Theorem 1.9

Proposition 4.1. For a given $\sigma_{0} \in(0,2)$, let

$$
\delta_{0}:=\min \left(\frac{\sigma_{0}}{2\left(2-\sigma_{0}\right)}, \frac{1}{2}\right)
$$

For $\sigma \in\left[\sigma_{0}, 2\right)$, let $l_{\sigma}$ be defined as (21). Then $l_{\sigma}$ satisfies Properties 1.1] and 1.2 with uniform constants $a_{0}, a_{\infty} \geq 1$ and $\rho \in(0,1)$ with respect to $\sigma \in\left[\sigma_{0}, 2\right)$, where the constants $a_{0}, a_{\infty}$ and $\rho$ depend only on $\sigma_{0}$, and the slowly varying function $l_{0}$.

Proof. Since $l_{0}$ varies slowly at zero and infinity, there exist $c_{0} \geq 1$ and $c_{\infty} \geq 1$ such that

$$
\begin{gathered}
\frac{l_{0}(s)}{l_{0}(r)} \leq c_{0} \max \left\{\left(\frac{s}{r}\right)^{\delta_{0}},\left(\frac{s}{r}\right)^{-\delta_{0}}\right\} \quad \forall r, s \in(0,1], \\
\frac{l_{0}(s)}{l_{0}(r)} \leq c_{\infty} \max \left\{\left(\frac{s}{r}\right)^{\delta_{0}},\left(\frac{s}{r}\right)^{-\delta_{0}}\right\} \quad \forall r, s \in[1,+\infty)
\end{gathered}
$$

from Potter's theorem; see Appendix $\mathrm{A}$ This implies that

$$
\frac{l(s)}{l(r)} \leq c_{0}^{2-\sigma} \max \left\{\left(\frac{s}{r}\right)^{-\sigma+\delta_{0}(2-\sigma)},\left(\frac{s}{r}\right)^{-\sigma-\delta_{0}(2-\sigma)}\right\} \quad \forall r, s \in(0,1],
$$

and

$$
\frac{l(s)}{l(r)} \leq c_{\infty}^{2-\sigma} \max \left\{\left(\frac{s}{r}\right)^{-\sigma+\delta_{0}(2-\sigma)},\left(\frac{s}{r}\right)^{-\sigma-\delta_{0}(2-\sigma)}\right\} \quad \forall r, s \in[1,+\infty) .
$$


By choosing $a_{0}:=c_{0}^{2}, a_{\infty}:=c_{\infty}^{2}$, and $\delta=\delta^{\prime}=\delta_{0}(2-\sigma)$, the property (a) in Property 1.1 and Property 1.2 hold since

$$
\delta=\delta^{\prime} \leq \frac{\sigma_{0}}{2\left(2-\sigma_{0}\right)}(2-\sigma) \leq \frac{\sigma_{0}}{2} \leq \frac{\sigma}{2} \quad \forall \sigma \in\left[\sigma_{0}, 2\right) .
$$

Lastly, the property (b) in Property 1.1 will be proved in the following Lemma4.2.

Lemma 4.2. Under the same assumption as in Proposition 4.1 we have that

$$
\frac{\sigma \int_{r}^{1} s^{-1} l_{\sigma}(s) d s}{l_{\sigma}(r)} \rightarrow 1 \quad \text { as } r \rightarrow 0+
$$

uniformly with respect to $\sigma \in\left[\sigma_{0}, 2\right)$. In particular, there exists a uniform constant $\rho \in$ $(0,1)$ such that for any $\sigma \in\left[\sigma_{0}, 2\right)$,

$$
\frac{1}{2} \leq \frac{\sigma \int_{r}^{1} s^{-1} l_{\sigma}(s) d s}{l_{\sigma}(r)}=\frac{L_{\sigma}(r)}{l_{\sigma}(r)} \leq 2, \quad \forall r \in(0, \rho)
$$

where $L_{\sigma}(r):=\sigma \int_{r}^{1} s^{-1} l_{\sigma}(s) d s$.

Proof. For $0<r<1$, we rewrite

$$
\frac{\int_{r}^{1} s^{-1-\sigma} l_{0}(s)^{2-\sigma} d s}{r^{-\sigma} l_{0}(r)^{2-\sigma}}=\int_{1}^{1 / r} t^{-1-\sigma}\left(\frac{l_{0}(t r)}{l_{0}(r)}\right)^{2-\sigma} d t .
$$

Note that for $t \in(1,1 / r)$

$$
\left(\frac{l_{0}(t r)}{l_{0}(r)}\right)^{2-\sigma} \leq c_{0}^{2-\sigma} t^{\delta_{0}(2-\sigma)}
$$

in the proof of Proposition 4.1. So the integrand is bounded by

$$
c_{0}^{2} t^{-1-\sigma+\delta_{0}(2-\sigma)}
$$

which is integrable since $\sigma-\delta_{0}(2-\sigma) \geq \sigma / 2 \geq \sigma_{0} / 2$. Thus it follows from the Dominated Convergence Theorem that $\int_{1}^{1 / r} t^{-1-\sigma}\left(\frac{l_{0}(t r)}{l_{0}(r)}\right)^{2-\sigma} d t$ converges to $\int_{1}^{+\infty} t^{-1-\sigma} d t=\frac{1}{\sigma}$ as $r \rightarrow 0+$ since $l_{0}$ varies slowly at zero. Now, it remains to show the uniform convergence with respect to $\sigma \in\left[\sigma_{0}, 2\right)$. Let $\varepsilon \in(0,1)$ be given. For a small uniform constant $r_{0} \in(0,1)$ to be chosen later, we have

$$
\begin{aligned}
\sigma \int_{1}^{1 / r} t^{-1-\sigma}\left(\frac{l_{0}(t r)}{l_{0}(r)}\right)^{2-\sigma} d t-1 & =\sigma \int_{1}^{1 / r_{0}} t^{-1-\sigma}\left\{\left(\frac{l_{0}(t r)}{l_{0}(r)}\right)^{2-\sigma}-1\right\} d t \\
& +\sigma \int_{1 / r_{0}}^{1 / r} t^{-1-\sigma}\left(\frac{l_{0}(t r)}{l_{0}(r)}\right)^{2-\sigma} d t-\sigma \int_{1 / r_{0}}^{\infty} t^{-1-\sigma} d t \\
& =: I_{1}+I_{2}+I_{3} .
\end{aligned}
$$

We select $r_{0}>0$ sufficiently small so that for $0<r<r_{0}$,

$$
\begin{aligned}
I_{2} & \leq \sigma c_{0}^{2} \int_{1 / r_{0}}^{1 / r} t^{-1-\sigma+\delta_{0}(2-\sigma)} d t=\sigma c_{0}^{2} \frac{1}{\sigma-\delta_{0}(2-\sigma)}\left\{r_{0}^{\sigma-\delta_{0}(2-\sigma)}-r^{\sigma-\delta_{0}(2-\sigma)}\right\} \\
& \leq \sigma c_{0}^{2} \frac{2}{\sigma} r_{0}^{\sigma / 2} \leq 2 c_{0}^{2} r_{0}^{\sigma_{0} / 2}<\frac{\varepsilon}{2}
\end{aligned}
$$

and hence

$$
\left|I_{3}\right|=\sigma \int_{1 / r_{0}}^{\infty} t^{-1-\sigma} d t \leq r_{0}^{\sigma} \leq r_{0}^{\sigma_{0} / 2}<\frac{\varepsilon}{4}
$$


Now we claim that for a fixed $r_{0}>0$,

$$
I_{1}:=\sigma \int_{1}^{1 / r_{0}} t^{-1-\sigma}\left\{\left(\frac{l_{0}(t r)}{l_{0}(r)}\right)^{2-\sigma}-1\right\} d t \rightarrow 0 \quad \text { as } r \rightarrow 0+
$$

uniformly with respect to $\sigma \in\left[\sigma_{0}, 2\right)$. According to the Uniform Convergence Theorem in [BGT, Theorem 1.5.2], we have that

$$
\frac{l_{0}(t r)}{l_{0}(r)} \rightarrow 1 \quad \text { as } r \rightarrow 0+\text { uniformly for } t \in\left[1,1 / r_{0}\right] .
$$

Then it follows that $\left(\frac{l_{0}(t r)}{l_{0}(r)}\right)^{2-\sigma}$ uniformly converges to 1 as $r \rightarrow 0+$ for $t \in\left[1,1 / r_{0}\right]$ and $\sigma \in(0,2)$. Namely, there exists a uniform constant $\rho \in(0,1)$ with respect to $\sigma \in\left[\sigma_{0}, 2\right)$, depending only on $l_{0}$ and $\sigma_{0}$, such that

$$
\left|\left(\frac{l_{0}(t r)}{l_{0}(r)}\right)^{2-\sigma}-1\right|<\frac{\varepsilon}{4}, \quad \forall r \in(0, \rho), t \in\left[1,1 / r_{0}\right] .
$$

Thus, we have that for any $0<r<\rho$,

$$
\begin{aligned}
\sigma \int_{1}^{1 / r_{0}} t^{-1-\sigma}\left\{\left(\frac{l_{0}(t r)}{l_{0}(r)}\right)^{2-\sigma}-1\right\} d t & <\frac{\sigma \varepsilon}{4} \int_{1}^{1 / r_{0}} t^{-1-\sigma} d t \\
& \leq \frac{\varepsilon}{4}\left(1-r_{0}^{\sigma}\right)<\frac{\varepsilon}{4}
\end{aligned}
$$

Therefore, for any $0<r<\rho$, we conclude that

$$
\left|\sigma \int_{1}^{1 / r} t^{-1-\sigma}\left(\frac{l_{0}(t r)}{l_{0}(r)}\right)^{2-\sigma} d t-1\right|<\varepsilon
$$

which finishes the proof.

\section{ApPendix A. Regular variations}

We recall regularly varying functions at zero and their properties. The results of regularly varying functions at infinity are established in $[\overline{\mathrm{BGT}}]$, which are simple inversions of those for regularly varying functions at zero.

Definition A.1 (Regular and slow variations). Let $l:(0,1) \rightarrow(0,+\infty)$ be a measurable function.

(i) A function $l:(0,1) \rightarrow(0,+\infty)$ is said to vary regularly at zero with index $\alpha \in \mathbb{R}$ if for every $k>0$

$$
\lim _{r \rightarrow 0+} \frac{l(\kappa r)}{l(r)}=\kappa^{\alpha} .
$$

(ii) A regularly varying function is called to be slowly varying if its index $\alpha$ is zero.

We state the important properties of regularly and slowly varying functions used in this paper as a lemma. The proofs and more details for regular and slow variations can be found in [BGT]; see also [KM] Appendix A].

Lemma A.2. Let $l:(0,1) \rightarrow(0,+\infty)$ be a measurable function.

(i) Any function l that varies regularly with index $\alpha \in \mathbb{R}$ is of the form

$$
l(r)=r^{\alpha} l_{0}(r)
$$

for some slowly varying function $l_{0}$. 
(ii) Let $l$ be a regularly varying function with index $-\alpha \leq 0$ which is locally bounded away from 0 and $+\infty$. Then Potter's theorem [BGT, Theorem 1.5.6] asserts that for any $\delta>0$, there exists $A_{\delta} \geq 1$ such that for $0<r, s<1$

$$
\frac{l(s)}{l(r)} \leq A_{\delta} \max \left\{\left(\frac{s}{r}\right)^{-\alpha+\delta},\left(\frac{s}{r}\right)^{-\alpha-\delta}\right\}
$$

(iii) Let $l$ be slowly varying and $\beta>-1$. Then Karamata's theorem [BGT, Proposition 1.5.8] asserts that

$$
\lim _{r \rightarrow 0+} \frac{\int_{0}^{r} s^{\beta} l(s) d s}{r^{\beta+1} l(r)}=\frac{1}{\beta+1} .
$$

(iv) Let l be a regularly varying function with index $-\alpha<0$. Then [BGT, Theorem 1.5.11] states that

$$
\lim _{r \rightarrow 0+} \frac{\int_{r}^{1} s^{-1} l(s) d s}{l(r)}=\frac{1}{\alpha} .
$$

This implies that if $l$ varies regularly with index $\alpha<0$, so does the function $r \mapsto$ $\int_{r}^{1} s^{-1} l(s) d s$.

(v) Let l be a regularly varying function with index $\alpha \in \mathbb{R}$. Then the Uniform Convergence Theorem [BGT, Theorem 1.5.2] asserts that

$$
\frac{l(\kappa r)}{l(r)} \rightarrow \kappa^{\alpha} \quad \text { as } r \rightarrow 0+\quad \text { uniformly in } \kappa \in[a, b]
$$

for each $[a, b] \subset(0,+\infty)$.

Acknowledgement The authors would like to thank Prof. Moritz Kassmann for suggesting the problem considered in this paper. Ki-Ahm Lee was supported by the National Research Foundation of Korea(NRF) grant funded by the Korea government(MSIP) (No.2014R1A2A2A01004618). Ki-Ahm Lee also hold a joint appointment with the Research Institute of Mathematics of Seoul National University.

\section{REFERENCES}

[A] S. Awatif, Équations d'Hamilton-Jacobi du premier ordre avec termes intégro-différentiels. I. Unicité des solutions de viscosité, Comm. Partial Differential Equations 16 (1991), 1057-1074.

[BI] G. Barles and C. Imbert, Second-order elliptic integro-differential equations: Viscosity solutions' theory revisited, Ann. Inst. H. Poincaré Anal. Non Linéaire 25 (2008), 567-585.

[BGT] N. H. Bingham, C. M. Goldie, and J. L. Teugels, Regular variation, Cambridge University Press, Cambridge, 1987.

[CC] L. Caffarelli and X. Cabré, Fully Nonlinear Elliptic Equations, American Mathematical Society Colloquium Publications, vol. 43, American Mathematical Society, Providence, RI, 1995.

[CS1] L. Caffarelli and L. Silvestre, Regularity theory for fully nonlinear integro-differential equations, Comm. Pure Appl. Math. 62 (2009), 597-638.

[CS2] L. Caffarelli and L. Silvestre, The EvansKrylov theorem for non local fully non linear equations, Annals of Mathematics 2 (2011), 1163-1187.

[DPV] E. Di Neza, G. Palatucci and E. Valdinoci, Hitchhikers Guide to the Fractional Sobolev Spaces, Bull. Sci. Math. 136 (2012), 521-573.

[J] R. Jensen, The Maximum Principle for viscosity solutions of fully nonlinear second order partial differential equations, Arch. Rat. Mech. Anal. 101 (1988), 1-27.

[KM] M. Kassmann and A. Mimica, Intrinsic scaling properties for nonlocal operators, arXiv: 1310.5371

[KSV] P. Kim, R. Song, Z. Vondraĉek, Potential Theory of Subordinate Brownian Motions Revisited, arXiv:1102.1369

[KL1] Y.C. Kim and K.A. Lee, Regularity results for fully nonlinear integro-differential operators with nonsymmetric positive kernels, Manuscripta mathematica 139 (2012), 291-319.

[KL2] Y.C. Kim and K.A. Lee, Regularity results for fully nonlinear integro-differential operators with nonsymmetric positive kernels: Subcritical Case. Potential Analysis 38 (2013), 433-455. 
[KL3] Y.C. Kim and K.A. Lee, Regularity results for fully nonlinear parabolic integro-differential operators, Mathematische Annalen 357 (2013), 1541-1576.

[KS] N. V. Krylov and M. V. Safonov, An estimate for the probability of a diffusion process hitting a set of positive measure, Dokl. Akad. Nauk SSSR 245 (1979), 18-20.

[LD1] H.C. Lara and G. Dávila, Regularity for solutions of nonlocal, nonsymmetric equations, Ann. Inst. H. Poincaré Anal. Non Linéaire 29 (2012), 833-859.

[LD2] H.C. Lara and G. Dávila, Regularity for solutions of non local parabolic equations, Calculus of Variations and Partial Differential Equations 49 (2014), 139-172.

Soojung Kim : National Institue for Mathematical Sciences, Daejeon, 306-390, Republic of Korea

E-mail address: soojung26@gmail.com; soojung26@nims.re.kr

Yong-Cheol Kim: Department of Mathematics Education, Korea University, Seoul 136-701, Republic of Korea

E-mail address: ychkim@korea.ac.kr

Ki-Ahm Lee: School of Mathematical Sciences, Seoul National University, Seoul 151-747, Republic of Korea \& Center for Mathematical Challenges, Korea Institute for Advanced Study, Seoul, 130-722, Republic OF KOREA

E-mail address: kiahm@math.snu.ac.kr 